Draft VERsion April 1, 2022

Preprint typeset using $\mathrm{AT}_{\mathrm{E}} \mathrm{X}$ style emulateapj v. 04/17/13

\title{
A NLTE ANALYSIS OF THE HOT SUBDWARF O STAR BD+284211. I. THE UV SPECTRUM
}

\author{
M. Latour ${ }^{1}$, G. Fontaine ${ }^{1}$, P. Chayer ${ }^{2}$, And P. Brassard ${ }^{1}$ \\ Draft version April 1, 2022
}

\begin{abstract}
We present a detailed analysis of the UV spectrum of the calibration star BD $+28^{\circ} 4211$ using highquality spectra obtained with the $H S T$ and FUSE satellites. To this aim, we compare quantitatively the observed data with model spectra obtained from state-of-the-art NLTE metal line-blanketed model atmospheres and synthetic spectra calculated with TLUSTY and SYNSPEC. We thus determine in a self-consistent way the abundances of eleven elements with well-defined lines in the UV, namely those of C, N, O, F, Mg, Si, P, S, Ar, Fe, and Ni. The derived abundances range from about solar to 1/10 solar. We find that the overall quality of the derived spectral fits is very satisfying. Our spectral analysis can be used to constrain rather tigthly the effective temperature of $\mathrm{BD}+28^{\circ} 4211$ to a value of $T_{\text {eff }}=82,000 \pm 5000 \mathrm{~K}$. We also estimate conservatively that its surface gravity falls in the range $\log g=6.2_{-0.1}^{+0.3}$. Assuming that the Hipparcos measurement for $\mathrm{BD}+28^{\circ} 4211$ is fully reliable and that our model atmospheres are reasonably realistic, we can reconcile our spectroscopic constraints with the available parallax measurement only if the mass of $\mathrm{BD}+28^{\circ} 4211$ is significantly less than the canonical value of $0.5 M_{\odot}$ for a representative post-EHB star.
\end{abstract}

Keywords: stars : atmospheres — stars : fundamental parameters — stars : individual $\left(\mathrm{BD}+28^{\circ} 4211\right)$ — subdwarfs

\section{INTRODUCTION}

$\mathrm{BD}+28^{\circ} 4211$ is a hot subwarf $\mathrm{O}(\mathrm{sdO})$ star whose brightness, high effective temperature, and relatively simple spectrum have made it a standard star in the optical domain as well as a calibration star for UV space missions such as IUE, HST, and FUSE. Its status of standard star implies that some of its observationnal properties are very well known. For example, high precision $U B V R I$ magnitudes have been presented by Landolt \& Uomoto (2007). In addition, its parallax mesurement in the Hipparcos catalogue places the star at $92_{-11}^{+14}$ pc. While studying $\mathrm{BD}+28^{\circ} 4211$ as a spectrophotometric standard, Massey \& Gronwall (1990) found it to have a faint red companion at a separation of $2.8^{\prime \prime}$. Moreover, BD $+28^{\circ} 4211$ has been extensively observed in the UV range by the missions mentionned above and there are highly valuable data available on that star. Ultraviolet spectra are precious tools for studying the chemical composition via the numerous metallic lines present in this wavelength range. It could be thought that with this privileged status, $\mathrm{BD}+28^{\circ} 4211$ would have been thoroughly studied and its physical parameters would be accurately known, but this is not exactly the case. In this connection, it has to be mentionned that because of its high effective temperature (around 80,000 $\mathrm{K}$ ), the local thermodynamic equilibrium (LTE) approximation is inappropriate for model atmospheres intended to represent the star. Instead, the more sophisticated and realistic approach of non-local thermodynamic equilibrium (NLTE) has to be used. Given the physical and technical difficulties associated with that approach, how-

\footnotetext{
${ }^{1}$ Département de Physique, Université de Montréal, Succ. Centre-Ville, C.P. 6128, Montréal, QC H3C 3J7, Canada; marilyn@astro.umontreal.ca

${ }^{2}$ Space Telescope Science Institute, 3700 San Martin Drive, Baltimore, MD 21218
}

ever, more efforts remain to be made along that avenue in order to characterize better the atmosphere of this star.

The first determination of the effective temperature of $\mathrm{BD}+28^{\circ} 4211$, using quantitative spectroscopy, has been made by Napiwotzki (1993) who estimated it to be around $82,000 \overline{\mathrm{K}}$. He determined this value by comparing the Balmer lines of the star with those of NTLE model atmospheres with a variable $\mathrm{H} / \mathrm{He}$ ratio (but with no metals) for different effective temperatures and surface gravities. This method is now largely used and its ability to give reliable fundamental parameters $\left(T_{\text {eff }}, \log \right.$ $g$, and sometimes also $N(\mathrm{He}) / N(\mathrm{H})$ ) for white dwarfs and hot subwarf B stars has already been demonstrated (Bergeron et al. 1992; Saffer et al. 1994). However, this method is rather tricky in the case of $\mathrm{BD}+28^{\circ} 4211$ because, like others sdO stars and white dwarfs that have high temperatures, the model spectra used generally suffer from the so-called Balmer line problem. That is, the observed lines cannot be simultaneously matched with a unique model spectrum. In other words, each line needs a model of different effective temperature in order to be well reproduced. Usually, the lowest lines in the series (like $\mathrm{H} \alpha$ and $\mathrm{H} \beta$ ) need a lower temperature, while the highest ones are better reproduced at higher temperatures. In the case of $\mathrm{BD}+28^{\circ} 4211$, to give the "extreme" values, $\mathrm{H} \alpha$ was best reproduced at $T_{\text {eff }} \simeq 50,000 \mathrm{~K}$ and $\mathrm{H} \epsilon$ at around $85,000 \mathrm{~K}$ (Napiwotzki 1993). The author also found a $\log g$ value of 6.2 and a solar helium abundance to be appropriate values for $\mathrm{BD}+28^{\circ} 4211$. His best estimate of the effective temperature of $\sim 82,000 \mathrm{~K}$ was subsequently confirmed by Dreizler \& Werner (1993) who checked that parts of the IUE UV spectrum of BD $+28^{\circ} 4211$, showing Fe VI and Fe VII lines, are properly reproduced at around 82,000 $\mathrm{K}$. This time, the NLTE model atmospheres they used included metals, namely carbon, oxygen, nitrogen, and 
iron group elements that were grouped together into 6 model atoms (one for each ionization stages between III and VIII). With similar models, and on the basis of the same IUE spectrum, Haas et al. (1996) estimated the abundance of iron to be about ten times subsolar while nickel was found to be nearly solar. According to them, oxygen and nitrogen also have abundances near the solar value. The improved UV spectra taken with STIS onboard the HST allowed to derived a solar abundance of manganese, while a sole line of chromium indicated an abundance between two and four times the solar value (Ramspeck et al. 2003).

The inclusion of metallic elements in NLTE model atmospheres, though costly in terms of computation time and complexity, allows not only for more realistic models, but also permits to solve, at least in part, the Balmer line problem (Werner 1996). We will address this issue in more details in the second paper of this serie (Latour et al. 2013, in preparation). The optical spectrum of $\mathrm{BD}+28^{\circ} 4211$ is rather featureless in comparison to its rich UV spectrum. Except for the Balmer and He II lines, nothing else is seen at medium resolution in the optical range. Because of this, $\mathrm{BD}+28^{\circ} 4211$ was chosen to be part of an investigation of diffuse interstellar bands in OB stars given its uncomplicated spectrum. However, the high resolution HIRES spectra of the star obtained for this investigation at the Keck I telescope show a lot of narrow absorption lines as well as a handful of emission lines (Herbig 1999). The sharpness of the absorption lines allowed to set an upper limit on the star's rotational velocity, $v \sin i \lesssim 4 \mathrm{~km} \mathrm{~s}^{-1}$, which is quite slow.

As for the high-quality FUSE spectrum of $\mathrm{BD}+28^{\circ} 4211$, it has only been used to study interstellar abundances in the line of sight of the star (Sonneborn et al. 2002). To our knowledge, this data set has not been exploited so far to better characterize the star. Although we have a general idea of its atmospheric chemical composition, no comprehensive or systematic studies were made on that star. With high-quality data available from $H S T$ and $F U S E$ in particular, we felt we should exploit them in order to reexamine the chemical composition of $\mathrm{BD}+28^{\circ} 4211$ and also try to constrain better its effective temperature and surface gravity by studying the ionization equilibrium of some metallic elements. Getting a portrait of the chemical composition of $\mathrm{BD}+28^{\circ} 4211$ should also be a first step in studying the optical spectrum of the star, with appropriate model atmospheres, for the Balmer line problem.

In the second section of this paper we describe our model atmospheres. This is followed by a short description of the observational material we used in Section 3, and of our abundance analysis in Section 4. We then discuss our attempts at constraining the effective temperature and the surface gravity by using the ionization equilibrium of metals in the UV range as well as the parallax distance in Section 5. Finally, we present a discussion and conclusion in Section 6.

\section{MODEL ATMOSPHERES}

\subsection{Characteristics of our Model Atmospheres}

We have developed the capacity to compute large grids of NLTE metal line-blanketed model atmospheres over reasonable timescales (days to weeks) with our paral- lel versions of TLUSTY and SYNSPEC that run on a dedicated cluster of computers (currently containing 320 processors). Our setup is described in more details in Latour et al. (2011) and has not changed since, apart from the increase in the number of processors we have available. Our final fully-blanketed model atmospheres for $\mathrm{BD}+28^{\circ} 4211$ include the following ions (besides those of $\mathrm{H}$ and $\mathrm{He}$ ) : $\mathrm{C}$ II to $\mathrm{C}$ V, $\mathrm{N}$ II to $\mathrm{N}$ VI, O II to $\mathrm{O}$ VII, $\mathrm{Si}$ III to $\mathrm{Si}$ V, $\mathrm{P}$ IV to $\mathrm{P}$ VI, $\mathrm{S}$ III to $\mathrm{S}$ VII, Fe IV to Fe VIII and Ni III to Ni VII. The highest ionization stage of each element is taken as a one-level atom. More information on the model atoms we used can be found on TLUSTY's web site ${ }^{3}$ and in Lanz \& Hubeny (2003, 2007). Since our thorough examination of $\mathrm{BD}+28^{\circ} 4211$ 's UV spectrum revealed also lines of argon, magnesium and fluorine, we needed to construct additional model atoms in order to study the abundance of these extra elements. This was done with the MODION program ${ }^{4}$ which uses the TOPBASE data (Lanz et al. 1996). This program allows the user to choose the explicit energy levels and also build superlevels that are included in the model atom. We thus constructed in this way model atons for the following ions : F III with 9 levels and 5 superlevels, F IV with 11 levels and 5 superlevels, F V with 19 levels and 3 superlevels, F VI with 12 levels and 5 superlevels, Mg III with 37 levels and 3 superlevels, Mg IV with 29 levels and 5 superlevels, Mg V with 18 levels and 2 superlevels, Ar IV with 39 levels, Ar V with 25 levels, Ar VI with 20 levels, and Ar VII with 18 levels. All transitions (bound-bound and bound-free) between these levels are thus considered when the ions are included in a model atmosphere.

Since Werner (1996) underlined the importance of using Stark profiles for CNO lines when modeling atmospheres of hot stars such as BD $+28^{\circ} 4211$, we inspected our different model atoms to check what kinds of profiles were used. We found that the strongest transitions (often resonance lines) of each ion are treated with Stark profiles while the weaker ones are represented by Doppler profiles. We then examined a synthetic spectrum that could represent $\mathrm{BD}+28^{\circ} 4211$ and identified its most prominent lines and made sure they were described with a Stark profile in the corresponding atomic model. This way we added a classic Stark profile to a few more lines of some elements, namely $\mathrm{C}$ IV, N IV, O IV and O V, Si IV and $\mathrm{P}$ V. A striking effect that Werner (1996) noticed when including Stark profiles was the disappearance of a high-temperature bump around $\log m$ of $-3 \mathrm{~g} \mathrm{~cm}^{-2}$ which was present when using Doppler profiles only (see his Figure 1). Since this bump is not seen either in our models (see our Figure 1), we believe our atomic data are appropriate for the study of $\mathrm{BD}+28^{\circ} 4211$ or other hot stars. Note that, even without modifying the original model atoms used by Lanz \& Hubeny (2003, 2007), our temperature structures do not present this bump (see Figure 4 of Latour et al. 2011).

The inclusion of our metallic elements must be done "step by step" if we want to assure the convergence of our models. Too drastic changes in the physical parameters of the model atmosphere used as input and the one we want to compute will prevent the latter from converging.

\footnotetext{
3 http://nova.astro.umd.edu/Tlusty2002/tlusty-framesdata.html

${ }^{4}$ http://idlastro.gsfc.nasa.gov/ftp/contrib/varosi/modion/README
} 


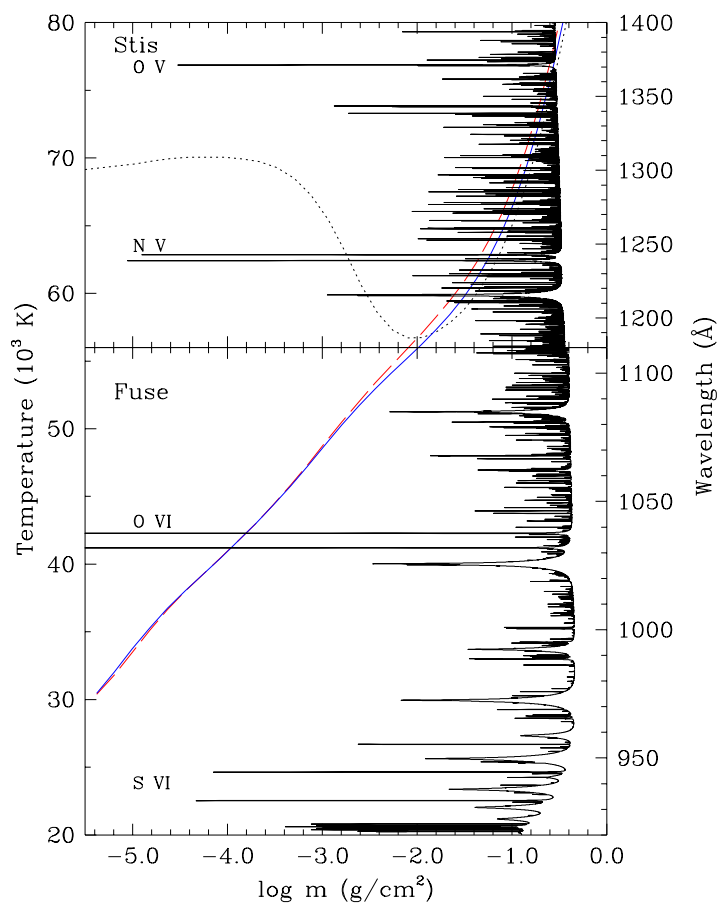

Figure 1. Temperature stratification and monochromatic optical depth $\tau_{\nu}=2 / 3$ as functions of depth, where $m$ is the column density, for NLTE models defined by $T_{\text {eff }}=82,000 \mathrm{~K}, \log g=6.2$, and $\log N(\mathrm{He}) / N(\mathrm{H})=-1.0$. The temperature structure is shown for models including $\mathrm{H}$ and $\mathrm{He}$ only (dotted curve), $\mathrm{H}, \mathrm{He}$, and $\mathrm{CNO}$ in solar abundances (solid curve), and $\mathrm{H}, \mathrm{He}$, and CNOSiPSFe in solar abundances (dashed curve). The $\tau_{\nu}=2 / 3$ curve is from the latter model and shows part of the FUSE and STIS wavelenght range in the UV.

Thus, when constructing a grid of these line-blanketed models, we end up with a number of "subgrids" including only some of the elements mentionned above. In the case of $\mathrm{BD}+28^{\circ} 4211$, we built five "subgrids" in order to end up with our final fully-blanketed one. For example, we have a grid including only $\mathrm{C}, \mathrm{N}$, and $\mathrm{O}$ in solar abundances, from which comes one of the models plotted in Figure 1.

In Figure 1, we show the temperature stratification for models with $T_{\text {eff }}=82,000 \mathrm{~K}, \log g=6.2$, and having a solar helium abundance culled from three of our grids. These estimates of the atmospheric parameters come from the work of Napiwotzki (1993). The first model is a "classical" pure $\mathrm{H}+\mathrm{He}$ NLTE model that shows the well known outwardly rise of temperature near the surface (dotted curve). The second one includes C, $\mathrm{N}$, and $\mathrm{O}$ (solid curve), while the third one includes all the elements of our final model (see above) besides nickel (dashed curve). At this point, all our elements have a solar abundance (Grevesse \& Sauval 1998). Though we plotted only three models in the figure, we examined the ones (having the same parameters) from our other grids and concluded that adding $\mathrm{S}, \mathrm{P}$ and $\mathrm{Si}$ to the $\mathrm{C}$, $\mathrm{N}$, O, only induces a minor drop of the temperature in the outer layers $(\log m<-2)$. When we add nickel to models already including $\mathrm{C}, \mathrm{N}, \mathrm{O}, \mathrm{Si}, \mathrm{P}, \mathrm{S}$, and $\mathrm{Fe}$, the changes in the temperature structure are unnotice- able on a graph like Figure 1. The cooling of the outer layers is thus mostly done by the inclusion of $\mathrm{C}, \mathrm{N}, \mathrm{O}$ elements ; adding more metals does not cool anymore the surface. As for the inner layers, their heating comes essentially from the presence of $\mathrm{C}, \mathrm{N}$, O, as well as $\mathrm{Fe}$ that was included afterward in the third model depicted in the figure.

It is easy to see from the temperature stratification that metallic elements, though they are not the dominant ones in the atmosphere, have an important effect on the thermodynamic structure at the surface of the star. Via their important opacity, they block a non-negligible part of the flux in the UV range, thus rising the continuum in the optical range. The presence of metals also causes a heating of the inner layers of the atmosphere, while it cools the outer layers. These changes in the atmospheric structure influence the emergent spectrum of the star, and thus the Balmer lines themselves. This is why their presence is an essential ingredient in the solution of the Balmer line problem (Werner 1996).

The other curve featured in Figure 1 shows the optical depth $\tau_{\nu}=2 / 3$ as a function of the column density $m$. It allows us to locate where the continuum and different lines are formed. The most opaque features, formed very near the surface, are the core of the resonance doublets of $\mathrm{S}$ VI, O VI and $\mathrm{N} \mathrm{V}$ as well as the $\mathrm{O} \mathrm{V}$ line at 1371 $\AA$. As for the broadest lines, they are either hydrogen or helium II ones. Depending on where a line is formed in the atmosphere, it might be affected differently by a change of the temperature structure.

In a model atmosphere with fundamental parameters like those of $\mathrm{BD}+28^{\circ} 4211$, some of the atomic species have a sole ion which dominates the atmosphere while the other ionization degrees have populations that are lower by orders of magnitude. This is the case for carbon, silicium and phosphorus, where ions in a noble gas configuration $(\mathrm{C} \mathrm{V}, \mathrm{Si} \mathrm{V}$ and $\mathrm{P} \mathrm{VI})$ are the ones that contribute the most to the thermodynamic structure of the atmosphere. The population of the other elements is dominated by ions of different ionization degrees depending on the depth in the atmosphere. We show the ionization equilibria of nitrogen and iron in Figure 2 for models including all the elements mentionned at the beginning of this section and having an effective temperature of $T_{\text {eff }}=82,000 \mathrm{~K}$ (solid lines) and $T_{\text {eff }}=92,000 \mathrm{~K}$ (dashed lines), a surface garvity $\log g=6.2$, and a solar helium content. The temperature is also shown in black for each model. Though the equilibrium changes as function of depth, $\mathrm{N} \mathrm{V}$ is dominant in the line forming region (around $\log m=-1$ ) while Fe VI and VII are in comparable proportions in this region for the model at $82,000 \mathrm{~K}$. However, when the temperature is raised to $92,000 \mathrm{~K}, \mathrm{Fe}$ VII become more dominant.

\subsection{A Word on the Inclusion of Metallic Elements}

By self-consistently including each element we want to study in our models, we allow it to influence the thermodynamical structure of the atmosphere. This is crucial if we want to get the right ionization equilibria and populations for our different ions. Dreizler \& Werner (1993) show that large discrepancies can arise in the strength of iron lines in a synthetic spectrum, depending on whether iron is included in a NLTE and self-consistent way or not. The inconsistent approach uses LTE statistics to 

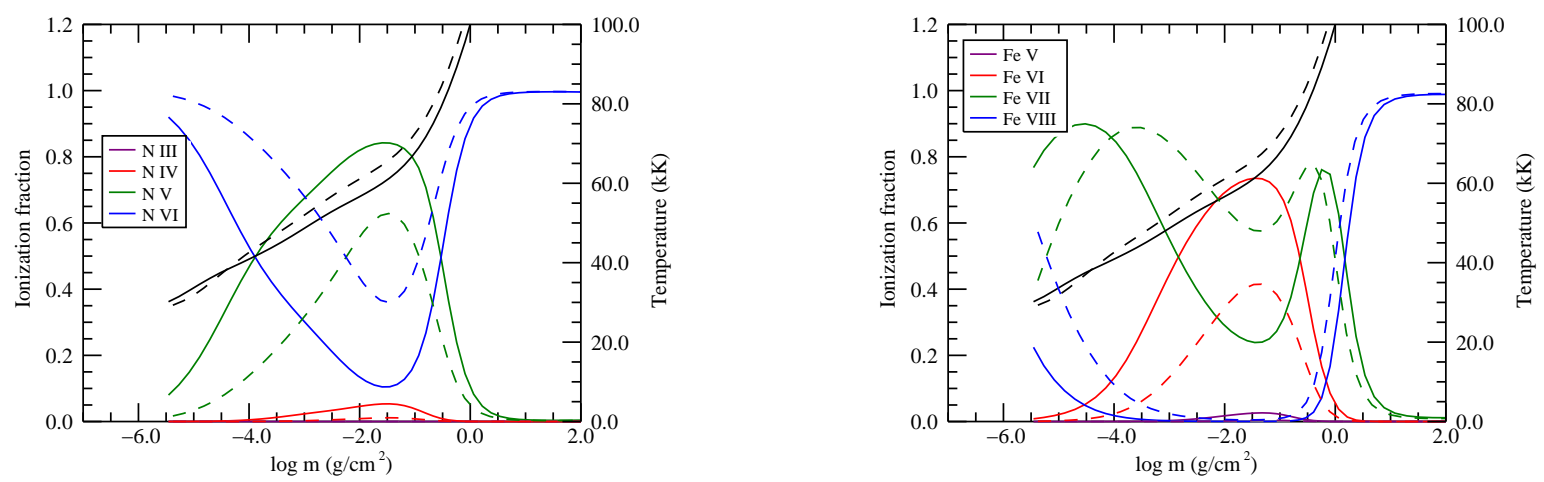

Figure 2. a) Ionization fractions of nitrogen as functions of depth in models with $T_{\text {eff }}=82,000 \mathrm{~K}$ (solid lines) and $92,000 \mathrm{~K}$ (dashed lines), $\log g=6.2$, and $\log N(\mathrm{He}) / N(\mathrm{H})=-1.0$. The black curves show the temperature profiles of the two models. b) Similar to Fig. 2a, but this time showing the ionization structure of iron.

obtain iron populations and ignore the back-reaction of the element on the atmospheric structure. The discrepancies increase between the two methods as the NLTE effects increase (as well as the abundance of the element in question), which means in our case, when the effective temperature increases. For BD $+28^{\circ} 4211$, it is essential that the elements we want to fit be consistently included in the models.

The TLUSTY package allows to add new elements afterward in the synthetic spectrum computed by SYNSPEC. Even though it is an inconsistent approach, we tried it just to take a look at some lines of additional elements we did not plan to include in our models because of their expected low abundances, the lack of model atoms, as well as the limit on the number of atomic levels and transitions the code can handle without becoming unstable. Even though for some atomic species we could easily make model atoms with the TOPBASE data, it is not the case for the iron-peak elements. They are not present in TOPBASE (except for iron) and their data (for iron and nickel) come from the Kurucz atomic data sets. Building model atoms for other elements of the iron peak would be a far more complex task than what was done for magnesium, fluorine, and argon. That being said, we added a solar amount of manganese, cobalt, and chromium in SYNSPEC to an already line-blanketed model atmosphere computed without these elements. When the resulting spectrum was compared with observations, it immediately appeared that there was a problem with the ionization equilibrium. Both $\mathrm{Mn}$ and $\mathrm{Cr}$ show lines of two differents ions ( $\mathrm{V}$ and $\mathrm{VI}$ ); lines corresponding to the higher ionization degree were well reproduced by our model while the ones from the lower degree were not correctly matched. In order to illustrate this, we included nickel in our synthetic spectrum with the determined abundance of this element (see section 4.1) and compared the result with our final model, which included nickel in the model atmosphere. We show in Figure 3 two regions of the STIS spectrum featuring some Ni V and VI lines. The two upper panels show the comparison between the observed spectrum of $\mathrm{BD}+28^{\circ} 4211$ and the synthetic one obtained by adding nickel $(\log N(\mathrm{Ni}) / N(\mathrm{H})=-6.0)$ in SYNSPEC only. The lower ones show the result of adding the same amount of nickel this time directly in the model atmosphere. In the case of nickel, Ni VI lines are definitely not strong enough in the upper panels, which is consistent with the observations of Lanz \& Hubeny (2003) that NTLE effects favor higher ionization degree due to the strong radiation field coming from the hotter and deeper layers that causes overionization. In the other hand, the $\mathrm{Ni} \mathrm{V}$ line shows in the second portion of the spectrum, as well as the few other we examined, remain quite unaffected by the difference in the inclusion method.

\section{ABUNDANCE ANALYSIS}

\subsection{Observational material \\ 3.1.1. FUV and $U V$ Observations}

A wealth of FUV and UV observations of BD $+28^{\circ} 4211$ has been gathered over the years. As we have mentioned earlier, BD $+28^{\circ} 4211$ has been used as a calibration star by several space observatories. In order to find all the FUV and UV observations of $\mathrm{BD}+28^{\circ} 4211$, we carefully searched the Mikulski Archive for Space Telescopes $(\mathrm{MAST})^{5}$. Spectroscopic observations of BD+28 4211 have been obtained not only with space observatories such as $I U E, F U S E$, and $H S T$, but also with instruments such as the Orbiting Retrievable Far and Extreme Ultraviolet Spectrometers and the Hopkins Ultraviolet Telescope that flew on space shuttle missions. Moreover, the $H S T$ spectroscopic observations include observations obtained with the Goddard High-Resolution Spectrograph and STIS. After retrieving the $\mathrm{BD}+28^{\circ} 4211$ data from MAST and looking at the available data, we selected the FUSE and STIS observations in order to carry out our spectroscopic analysis. The choice of these spectroscopic data is based on the wide wavelength range covered by both instruments, and on their higher resolution and higher signal-to-noise ratio than the data obtained by other instruments. The next two sections describe in detail the FUSE and STIS data that we selected for our analysis.

\footnotetext{
${ }^{5}$ http://archive.stsci.edu/index.html
} 
Table 1

STIS Datasets Retrieved From StarCAT

\begin{tabular}{lcccr}
\hline \hline \multicolumn{1}{c}{ Dataset } & Grating & $\lambda_{\text {cen }}(\AA)$ & Range $(\AA)$ & Exp. Time $(\mathrm{s})$ \\
\hline E140M-1425_020X020_50710-53135 & E140M & 1425 & $1140.5-1730.0$ & 20607 \\
E140H-1416_020X020_50812-53135 & E140H & 1416 & $1325.1-1505.8$ & 15311 \\
E230M-1978_020X020_50710-53135 & E230M & 1978 & $1611.1-2150.1$ & 9510 \\
E230H-2263_020X020_50812-53135 & E230H & 2263 & $2139.5-2394.0$ & 22962 \\
E230H-2513_020X020_52461 & E230H & 2513 & $2386.5-2639.6$ & 2674 \\
E230M-2707_020X020_50678-53135 & E230M & 2707 & $2629.6-3118.2$ & 22547
\end{tabular}

\subsubsection{FUSE Observations}

FUSE covers a wavelength range of $905 \AA$ to $1187 \AA$ with a resolution of about $R=\lambda / \Delta \lambda=20,000$. For more information about the design of the instrument and the spectroscopic data products, see Moos et al. (2000), Sahnow et al. (2000), and Dixon et al. (2007). During the lifetime of the FUSE mission, several observations of $\mathrm{BD}+28^{\circ} 4211$ have been carried out under three calibration programs. The first FUSE observation of $\mathrm{BD}+28^{\circ} 4211$ was obtained through the program M108 in order to establish the suitability of the star to the calibration program, given that its FUV flux was close to the bright limit of the FUSE detectors. Subsequently, $\mathrm{BD}+28^{\circ} 4211$ was observed under the program M104 to test the use of the focal plane splits (FP splits) in order to mitigate the fixed-pattern noise, and therefore to increase the $\mathrm{S} / \mathrm{N}$ ratio of the final spectrum. This technique consisted in taking a series of exposures at dif-

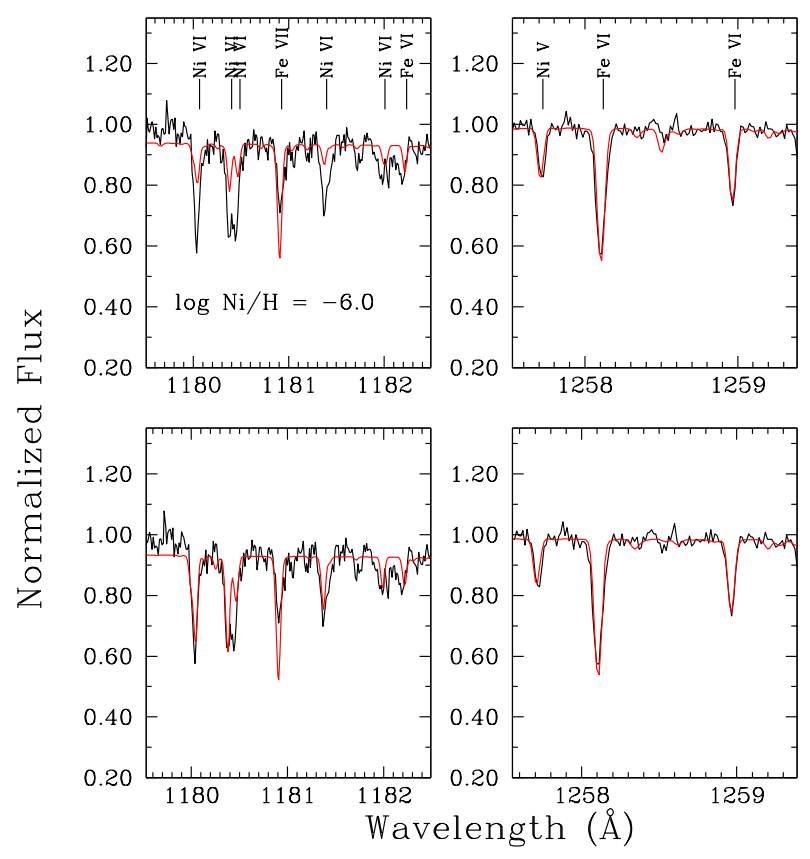

Figure 3. Comparison between portions of the observed STIS spectrum of $\mathrm{BD}+28^{\circ} 4211$ and synthetic spectra. The two upper panels show the synthetic spectra obtained when nickel is included in the spectrum only, thus in an inconsistent manner. In the lower panels, nickel was included from the beginning in the model atmosphere. It is possible to see the differences arising from the two ways of treating the presence of nickel in the models. ferent focal plane assembly positions. In this way, the corresponding spectra were shifted along the dispersion direction and exposed on different portions of the detectors. The fixed-pattern noise was therefore reduced by co-aligning and co-adding all the spectra. The M104 observations consisted of exposures using the LWRS and MDRS apertures. Finally, two series of short observations taken through the LWRS, MDRS, HIRES apertures were carried out under the M103 program, which monitored the photometric stability of the FUSE instrument.

We selected the M1080901, M1040101, M104105, M1031201, and M1031204 observations. All these observations were taken through the LWRS aperture and recorded in spectral image mode, or histogram (HIST) mode. We considered 66 exposures with an average exposure time of about $485 \mathrm{~s}$, such that the total exposure time amounts to about $32,000 \mathrm{~s}$. The eight FUSE segments, LiF1A, LiF1B, LiF2A, LiF2B, SiC1A, SiC1B, $\mathrm{SiC} 2 \mathrm{~A}$, and $\mathrm{SiC} 2 \mathrm{~B}$ were cross-correlated and co-added. All LiF1B exposures show a depression in flux between $1130 \AA$ and $1170 \AA$. This depression in flux is caused by a electron repeller wire grid that can cast shadows on the detectors. The LiF1B data in this wavelength range were not considered. A final co-added FUSE spectrum was obtained by considering and merging the following spectral regions: $\operatorname{SiC} 1 B(905-990 \AA)$, LiF1A (990-1080 $\AA), \mathrm{SiC} 2 \mathrm{~B}(1080-1090 \AA)$, LiF2A $(1090-1180 \AA)$, and LiF1B (1180-1187 $\AA$ ). The final spectrum has a signalto-noise ratio $\mathrm{S} / \mathrm{N} \sim 68$ at $950 \AA, \mathrm{S} / \mathrm{N} \sim 130$ at $1050 \AA$, and $\mathrm{S} / \mathrm{N} \sim 89$ at $1150 \AA$.

The FUSE spectrum displays many interstellar and stellar absorption lines. The most prominent stellar lines are the Lyman series of hydrogen starting from $\mathrm{Ly} \beta$ up to Ly-8. There are followed by the He II lines that involve transitions between energy levels $n_{l}=2 \rightarrow n_{u}=4$ up to 13. Transitions with upper even numbers produce, however, lines that are blended with the hydrogen Lyman lines. The strongest metal lines are the O VI $\lambda \lambda 1031.91$ and 1037.61 resonance lines that have equivalent widths of about $400 \mathrm{~m} \AA$ each. It is interesting to note that the O VI doublet does not display any P Cygni profile characteristic, which suggests that no stellar wind expanding away from $\mathrm{BD}+28^{\circ} 4211$ is detected. In addition to the O VI lines, many metal lines of highly ionized species are observed. For instance, the following ions are observed in the FUSE spectrum: C IV, N IV, O IV, O V, F IV, F VI, Si IV, P V, S IV, S V, S VI, Ar VII, Fe VI, Fe VII, Co VI, and Ni VI. The equivalent widths of the metal lines range from a few $\mathrm{m} \AA$ to $\sim 150 \mathrm{~m} \AA$. Although a large number of metal lines have been identified, there are still about 150 absorption features with no identifica- 
tion. This state of affairs is also observed in FUV and UV spectra of many hot stars, and reflects the lack of atomic data for highly ionized species. Finally, the FUSE spectrum of $\mathrm{BD}+28^{\circ} 4211$ contains a large number of strong absorption lines from interstellar $\mathrm{H}_{2}$, and also several absorption lines from species such as H I, D I, C I, C II, N I, O I, P II, Ar I, and Fe II. Sonneborn et al. (2002) analyzed the line of sight toward BD $+28^{\circ} 4211$ by using FUSE and STIS data, and measured interstellar D I, H I, $\mathrm{N}$ I, O I, Fe II and $\mathrm{H}_{2}$ column densities.

\subsubsection{STIS Observations}

Since the installation of STIS on board HST in 1997, $\mathrm{BD}+28^{\circ} 4211$ has been observed to monitor the sensitivity of each Multi-Anode Microchannel Array (MAMA) echelle grating mode. Although STIS suffered a major failure in August 2004, it returned to science operations after being repaired during the fourth $H S T$ servicing mission in May 2009. To this date about 206 observations of $\mathrm{BD}+28^{\circ} 4211$ have been carried out with the echelle gratings E140M and E140H FUV-MAMA, and the E230M and E230H NUV-MAMA. Specifically, BD+28 4211 has been observed with the following echelle grating setups: E140M (central wavelength of spectrum $\lambda_{\text {cen }}=1425 \AA$;

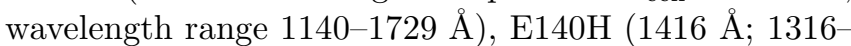

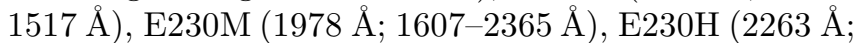

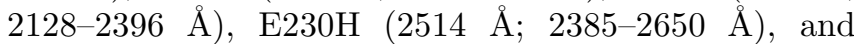
E230M (2707 $\AA ; 2275-3118 \AA$ ). Therefore the spectroscopic coverage ranges from $1140 \AA$ to $3118 \AA$. The resolution $R=\lambda / \Delta \lambda$ for the E140M and E230M is $\sim 45,800$, and it is $\sim 114,000$ for the $\mathrm{E} 140 \mathrm{H}$ and $\mathrm{E} 230 \mathrm{H}$. All the observations were carried out by using the $0.2^{\prime \prime} \times 0.2^{\prime \prime}$ aperture. The exposure times for the individual observations are on average $\sim 360 \mathrm{~s}$ and $\sim 500 \mathrm{~s}$ for the E140M and E230M observations, and they are $\sim 1000 \mathrm{~s}$ and $\sim 1500 \mathrm{~s}$ for the $\mathrm{E} 140 \mathrm{H}$ and $\mathrm{E} 230 \mathrm{H}$ observations.

Instead of using all the STIS observations of $\mathrm{BD}+28^{\circ} 4211$ that are available at MAST, we opted to retrieve the observations from StarCAT $^{6}$, which is a STIS echelle spectral catalog of stars that Ayres (2010) created based on observations of high-resolution spectra. StarCAT contains all the echelle high-resolution spectra of $\mathrm{BD}+28^{\circ} 4211$ that were collected from 1997 to the failure of STIS in 2004. Given that BD $+28^{\circ} 4211$ has been observed frequently, Avres (2010) cross-correlated and coadded all the $\mathrm{BD}+28^{\circ} 4211$ spectra in order to achieve a high signal-to-noise ratio. We retrieved six datasets from Avres (2010)'s StarCAT. The properties of the datasets are summarized in Table 1. The table gives the name of the dataset, the grating that was used with its setting $\lambda_{\text {cen }}$, the wavelength range, and the total exposure time in seconds that is the sum of all the exposures. The resulting signal-to-noise ratio for the observations taken with the E140M at $\lambda_{\text {cen }}=1425 \AA$ is $\mathrm{S} / \mathrm{N} \sim 180$ at $1250 \AA$ and $\mathrm{S} / \mathrm{N} \sim 145$ at $1500 \AA$. The observations performed with the $\mathrm{E} 230 \mathrm{H}$ at $\lambda_{\text {cen }}=2513 \AA$ has the shortest exposure time and consequently has the lowest signal-to-noise ratio with $\mathrm{S} / \mathrm{N} \sim 30$ at $2500 \AA$. The remaining observations have signal-to-noise ratios greater than 100 . The resulting STIS spectra of $\mathrm{BD}+28^{\circ} 4211$ with their high signal-to-noise ratio and large spectral coverage are FUV and UV data of outstanding quality.

As in the case of the FUSE data, the STIS data show a large number of absorption lines. The greatest number of lines are observed between $1140 \AA$ and $\sim 1475 \AA$. Beyond $1475 \AA$, the intensity and the number of lines decrease dramatically. On the short wavelength side, absorption lines of high-ionization species such as Fe V, Fe VI, Fe VII, Co VI, Ni V, and Ni VI are the most numerous. The equivalent widths of these absorption lines vary from a few $\mathrm{m} \AA$ to $\sim 75 \mathrm{~m} \AA$. The strongest line observed in the STIS spectrum is Ly $\alpha$. In fact, most of the absorption at Ly $\alpha$ comes from the interstellar $\mathrm{H}$ I along the line of sight of BD $+28^{\circ} 4211$. Sonneborn et al. (2002) measured a $\mathrm{H}$ I column density of $\log N(\mathrm{H} \mathrm{I})=19.842$ in the direction of $\mathrm{BD}+28^{\circ} 4211$, and showed that the stellar H I component is much fainter than the interstellar absorption. The second strongest observed line is the He II $\lambda 1640$ line $\left(n_{l}=2 \rightarrow n_{u}=3\right)$ that has an equivalent width of about $1200 \mathrm{~m} \AA$. The line shows broad wings that extend to about $4.5 \AA$ from the center of the line, and it shows a shallow core. Interestingly, the He II line series $n_{l}=3 \rightarrow n_{u}=6$ up to 11 is observed at longer wavelengths. The wings of these He II lines are also broad, but the lines show a much shallower core than the He II $\lambda 1640$ line. The $\mathrm{N}$ V $\lambda \lambda 1238,1242$ lines and $\mathrm{O} V \lambda 1371$ line are the strongest metal lines observed in the STIS spectrum. The lines have equivalent widths of about $770 \mathrm{~m} \AA, 482 \mathrm{~m} \AA$, and $574 \mathrm{~m} \AA$. The total equivalent widths of the Si IV $\lambda \lambda 1393,1402$ lines and C IV $\lambda \lambda 1548$ and 1550 lines have equivalent widths of about $125 \mathrm{m \AA}$ and $340 \mathrm{m \AA}$.

Even though we have identified most stellar and interstellar absorption lines in the STIS spectrum of $\mathrm{BD}+28^{\circ} 4211$, there are still about 260 lines that do not have any identification. The equivalent widths of these lines range from a few $m \AA$ to a few ten of $m \AA$. The total approximate number of lines with no identification in both FUSE and STIS spectra is around 410 lines. We extended our search of metal lines to elements beyond Zn. For instance, O'Toole \& Heber (2006) and Chaver et al. (2006) observed strong absorption lines of heavy elements such as $\mathrm{Ga}, \mathrm{Ge}, \mathrm{Zr}, \mathrm{Sn}$, and $\mathrm{Pb}$ in FUSE and STIS spectra of sdB stars, while Vennes et al. (2005) and Chayer et al. (2005) observed Ge and Sn in a handful of hot DA white dwarfs, and Ge, As, Se, Br, Sn, Te, and I in two cool DO white dwarfs. Werner et al. (2012) added the discovery of $\mathrm{Kr}$ and $\mathrm{Xe}$ in the atmosphere of the DO white dwarf RE $0503-289$ to the list of heavy elements detected in the atmospheres of compact stars. We looked for these heavy elements in both FUSE and STIS spectra by using the wavelengths of the high-ionization species. We also added Mo to our search. Unfortunately, no lines from heavy elements are observed. There is an absorption feature around $987.6 \AA$ that could correspond to the As V $\lambda 987$ line, but no absorption feature matches the second component of the As V doublet at $1029.48 \AA$ adequately. As we have concluded in the previous section, the non-identification of many absorption features in the FUSE and STIS spectra of $\mathrm{BD}+28^{\circ} 4211$ illustrates the lack of atomic data of high-ionization species.

\footnotetext{
${ }^{6}$ http://archive.stsci.edu/prepds/starcat/
} 


\subsection{Fitting Technique and Resulting Abundances}

Our fitting technique consists in minimizing a $\chi^{2}$-type value defined as the sum of the squared difference between the model and observed fluxes over a given range of wavelength. Our free parameters are the solid angle and, obviously, the abundance of the fitted element. It is also possible to add a trend (linear or quadratic) if the continuum in the range of interest needs one, which was sometimes useful since the normalized UV spectra are not always as flat as they should.

The method used to determine the abundance is a partial iterative one. First of all, from the work of Napiwotzki (1993), the temperature and gravity of our models are held fixed at $T_{\text {eff }}=82,000 \mathrm{~K}$ and $\log g=6.2$, and the helium abundance is fixed at the solar value. Most of our metal grids consist of six or seven different abundances centered around the solar one and varying in steps of 0.5 dex. For example, in the case of oxygen (whose solar abundance is $\log N(\mathrm{O}) / N(\mathrm{H})=-3.3$ ), its grid covers a range from $\log N(\mathrm{O}) / N(\mathrm{H})=-2.0$ to -4.5 in steps of 0.5 dex. Since we have a better idea of the iron and nickel abundances (thanks primarily to the work of Ramspeck et al. (2003)), their grid meshes are narrower ; between one tenth and one time solar for iron, and centered around the solar value for nickel, but this time varying only in steps of 0.2 dex. In the first step of the procedure, we used models including $\mathrm{C}, \mathrm{N}, \mathrm{O}$, and $\mathrm{Fe}$ in solar abundances initially and then varied the abundances of these four elements, one at a time. We then used these improved models to build the grids for silicon, sulfur, and phosphorus. We were then able to obtain a first estimate of the abundances for these seven elements. The number of lines fitted for each element is given in Table 2. We carefully chose lines that are reasonably well isolated in regions of the spectrum not too crowded with blends or interstellar lines. Our second step was to redo our grids for each element, this time including the previously found abundances for the other elements. At this point, when redoing our fitting procedure, the values found for individual lines sometimes changed a little, but the mean abundances stayed roughly the same. Once we got the abundances of C, N, O, Si, P, S, and Fe fixed, we made new grids in order to obtain, this time, a value for nickel, fluorine, magnesium, and argon. The new grids for these four elements only included, besides the element of interest, $\mathrm{C}, \mathrm{N}, \mathrm{O}$ and $\mathrm{Fe}$, which are the atomic species playing the major role in setting up the atmospheric structure. We took a particular interest in argon because the spectrum of $\mathrm{BD}+28^{\circ} 4211$ features two strong lines of this element, coming from two ionization states : Ar VI at $1303.86 \AA$ and Ar VII at $1063.63 \AA$.

After these three steps, we were able to draw up a good portrait of the chemical composition of $\mathrm{BD}+28^{\circ} 4211$, with quite satisfactory fits. Our final results are summarized in Table 2. The first column shows the different elements as well as the ionic species present in our sample of fitted lines. We then give the number of fitted intervals (like the six ones presented in Figure 4) and the total number of lines (from the element of interest) included in those intervals. The third column presents the mean abundance of the analyzed ranges while the fourth one gives the standard deviation associated with the previous column. Finally, the last one gives the total uncertainty of our abundances, which will be discussed in the next subsection.

We tentatively tried to formally fit some fluorine lines, but since they were either too faint, or blended the results were not conclusive. Our result is thus based on a sole isolated line, F IV $\lambda 1059.719$, for which we visually estimated an abondance of $\log N(\mathrm{~F}) / N(\mathrm{H})=-8.0$ to be appropriate. The resulting comparison can be found in the last panel of the online Figure. This result is compatible with the other lines we checked $(\lambda \lambda$ 1082.345, 1088.400 and 1139.523), although these lines are either blended or in a noisy region of the spectrum for which their faintness does not help.

For the sake of completeness, we also examined the chromium, manganese and cobalt lines visible in our spectra and estimated their abundances. This time we had to add these elements afterward in the synthetic spectrum (as explained in section 2.2) where their populations were computed assuming LTE. The model atmosphere used as input for the spectra computation included our main metallic elements (C, N, O, Si, P, S, $\mathrm{Fe}$, and $\mathrm{Ni}$ ). The abundances of the three studied elements were in turn varied in the synthetic spectra and then fitted the same way we did for the other elements. In order to avoid the ionization problem discussed in section 2.2, we fitted only lines coming from the dominant ionization stage, which is VI for these three iron-peak elements. As for the previously fitted elements, the uncertainties include the standard deviation as well as effects from a change of temperature and surface gravity in the input model atmosphere. Despite the fact that our resulting abundances presented in Table 3 are only rough estimates, obtained with an approximate method, our values for $\mathrm{Cr}$ and $\mathrm{Mn}$ are in good agreement with the ones found by Ramspeck et al. (2003), which were around -5.88 for $\mathrm{Cr}$ and -6.62 for $\mathrm{Mn}$.

Figures 4 and 5 show a sample of our fitted intervals taken from the STIS (Figure 4) and FUSE (Figure 5, except the top left panel) spectra. The abundance resulting from the fitting procedure of the featured region is given as $\log N(\mathrm{X}) / N(\mathrm{H})$. We show here the final step of our fitting method, so the elements included, besides the fitted one, have abundances corresponding to the ones presented in Table 2. The totality of our fitted spectral chunks are available as online material.

Some of our intervals include important interstellar lines, such as $\mathrm{O}$ I, $\mathrm{N}$ I, $\mathrm{C}$ I, $\mathrm{H} \mathrm{I}$ and $\mathrm{H}_{2}$. Interstellar shortward-shifted $\mathrm{C}$ IV and $\mathrm{Si}$ IV components are also seen for both doublets lines (one of which is shown in Figure 4). These features are thought to originate from a circumstellar cloud or shell near the star (Bruhweiler \& Dean 1983). Since BD+284211 has some similarities with central stars of old planetary nebulae in its spectrum as well as in its fundamental parameters, this could be some sort of old planetary nebula remnant. When strong ISM lines are present in our fitted intervals, we exclude them from our minimization procedure by iteratively rejecting wavelength points of the observed spectrum too far from the model's ones.

We carefully inspected each fitted range to make sure the resulting model was appropriate, the continuum was at a satisfactory level, and to notice any discrepant lines. Our checkup highlighted some points we found worth 

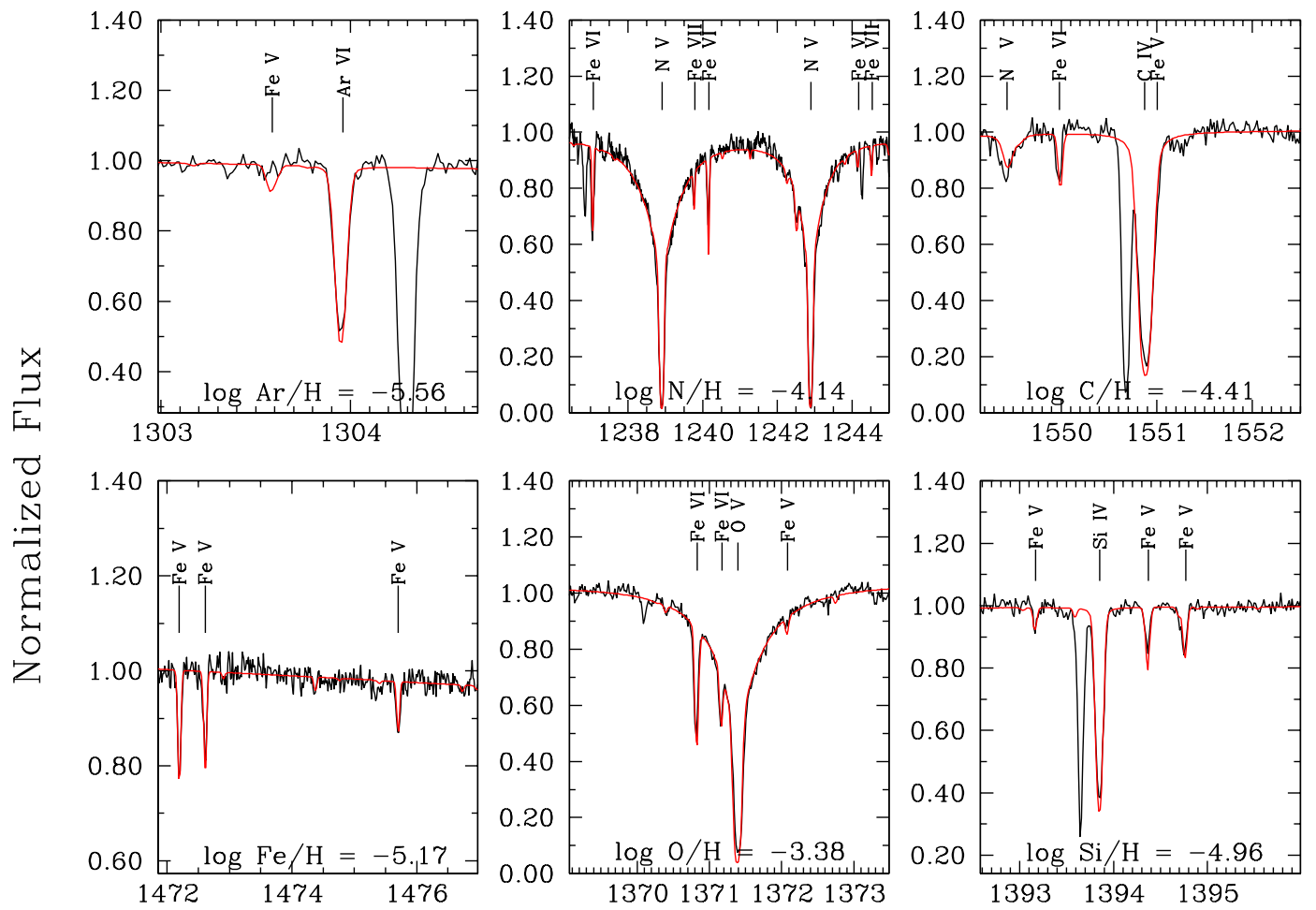

Wavelength $(\AA)$

Figure 4. Sample of our fitted spectral intervals from the STIS spectrum. The red curve shows the result of the fitting procedure for the element mentionned at the bottom of each panel, where the resulting abundance for the interval is expressed as $\log N(\mathrm{X}) / N(\mathrm{H})$. All our models have $T_{\text {eff }}=82,000 \mathrm{~K}, \log g=6.2$, and $\log N(\mathrm{He}) / N(\mathrm{H})=-1.0$. Interstellar features are visible in some spectral chunks, such as a strong Si II line beside Ar VI $\lambda 1303$ and shortward-shifted C IV and Si IV lines.

mentionning.

First of all, if we consider the most prominent features (i.e., resonance and strong lines), they are all well reproduced. The only exception is the O VI doublet $(\lambda \lambda 1032$, 1038), for which the cores happen to be too opaque in our models. This phenomenon can also be seen, at a smaller amplitude, in the cores of the resonance doublet of S VI $(\lambda \lambda 933,944)$, in $\mathrm{S}$ vi $\lambda 1117.76$ and in $\mathrm{O} v \lambda 1371.3 . \mathrm{We}$ have to mention here that the analysis of Rauch et al. (2007) has been a useful reference to us on this particular account. They analyzed STIS and FUSE spectra of a star (LS V $+46^{\circ} 21$ ) whose fundamental parameters $\left(T_{\text {eff }}=95,000 \mathrm{~K}\right.$ and $\left.\log g=6.9\right)$ give it a spectrum quite similar to that of $\mathrm{BD}+28^{\circ} 4211$, in the sense that both star show lots of common lines. It allows us to compare some of our fits with theirs. This way, we noticed that they reported the same effect of too strong cores in resonance lines of their star, letting us know the issue is not only about our own model atmospheres.

Secondly, when choosing our sample of lines to be fitted, a few lines gave inconsistent results or could not be matched in any way whatsoever. For instance, our attempt to fit the O IV structure around $1081 \AA$ was not conclusive enough to be included in our final sample because the core of the central component at 1080.97 $\AA$ was too strong in our models. The N IV triplet between
1225 and $1226 \AA$ required a much higher abundance (log $N(\mathrm{~N}) / N(\mathrm{H})=-3.2)$ than our other lines of nitrogen, so we did not include it our sample. The same thing happened for $\mathrm{O} v \lambda 968.9$, but this time we suspect this line to be blended with interstellar $\mathrm{H}_{2}$ lines at 968.997 and/or $969.07 \AA$. This possibility is supported by the few $\mathrm{H}_{2}$ lines present in the vicinity of the oxygen line.

Finally, some odd lines of oxygen appear in our models, whereas there is no sign of them in the observed spectrum. We noticed $\mathrm{O}$ III $\lambda 1153.775$ and two $\mathrm{O}$ IV lines at 1076.06 and $1294.065 \AA$. A too strong oscillator strength value might be the cause of their presence. We should also mention four other lines, among which three of them appear also too strong in our final model, namely $\mathrm{Fe} \mathrm{V}$ $\lambda 1393.072$ and Fe VII $\lambda \lambda 1154.990,1180.827$. As for the last one, Ni VI at $1204.078 \AA$ is too faint in our models and was excluded from our fitted nickel sample. However, these lines also appear too strong (or too faint for the nickel one) in the final model of Rauch et al. (2007), so there is a strong chance that the oscillator strengths of the lines are involved here.

\subsection{Evaluation of the Abundance Uncertainties}

Hot stars sometimes "become" hotter with time, in the sense that their estimated effective temperature tend to be revised upwards. For example, the DAO white dwarf 

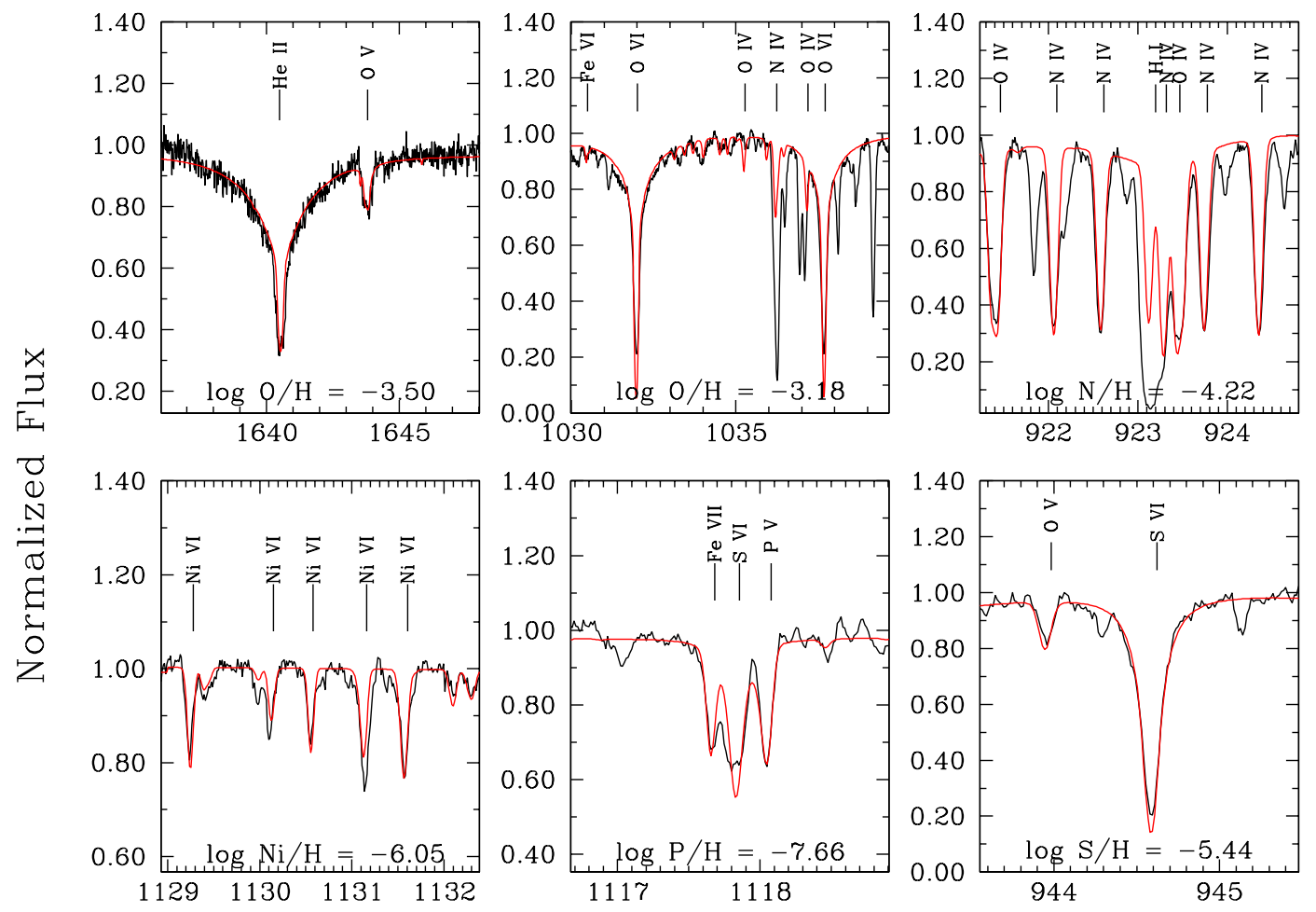

Wavelength $(\AA)$

Figure 5. Same as Figure 4 but for parts of the FUSE spectrum, except for the first interval, showing He II $\lambda 1640$, which comes from the STIS spectrum. Note the He II line which is particularly well reproduced with the assumed fundamental parameters. There is a lot more interstellar lines in the FUSE wavelength range. We notice in this figure, among others, C II $\lambda 1036.3$, O I $\lambda \lambda 1039.19$, 921.82 and C I $\lambda 945.15$.

LS V $+46^{\circ} 21$, has seen its estimated effective temperature go from $83,000 \mathrm{~K}$ in Napiwotzki (1999) to 95,000 $\mathrm{K}$ in the thorough study of Rauch et al. (2007). In a most extreme case, the white dwarf KPD 0005+5106 was shown to be the hottest known DO in Werner et al. (1994) with an estimated value of $T_{\text {eff }}=120,000 \mathrm{~K}$, but a subsequent analysis of better spectroscopic data found lines of highly ionised metals (Ne VIII and $\mathrm{Ca} \mathrm{X}$ ), thus needing the star to have an effective temperature of at least $180,000 \mathrm{~K}$ (Wassermann et al. 2010). With these rather extreme cases in mind, we wanted to have an idea of how our abundances would change if the effective temperature or the surface gravity of $\mathrm{BD}+28^{\circ} 4211$ end up being different from our assumed values.

Therefore, we redid a part of our abundance analysis, using in a first step models with an effective temperature of $92,000 \mathrm{~K}$, and then models at $\log g=6.6$. To do this for all the atomic species studied in the previous section would require computing twice as many grids as we did for determining the abundances themselves. We therefore decided to do this exercise for three elements only, namely nitrogen, silicon, and iron. The resulting abundances are presented in Table 4 which gives, for each model considered, the abundance and standard deviation obtained for the indicated element. The first column specifies the model used in our fitting procedure by indicating the parameter that has been changed with respect to the ones used in the previous section. The first quoted model is our reference model at $T_{\text {eff }}=82,000$ $\mathrm{K}$ and $\log g=6.2$. One thing to note here is that a change in the effective temperature of the models of the magnitude considered here induces a larger change in the determined abundance than a change in $\log g$. Another interesting thing to look at is the value of $\sigma$, the standard deviation of the different spectral chunks we fitted. In the case of iron and nitrogen, this value is larger for $T_{\text {eff }}=92,000 \mathrm{~K}$ and $72,000 \mathrm{~K}$, meaning the abundances obtained from each interval agree less with each other than in the reference case. The results for silicon are somewhat different because there is only three fitted lines, produced by a sole ion (Si IV), whereas nitrogen and iron have lines produced by two and three ions. Silicon is thus less expected to show larger discrepancies between its fitted lines at different atmospheric parameters. The behavior of the standard deviation (for nitrogen and iron) with the temperature can be taken at a reassuring sign pointing towards $82,000 \mathrm{~K}$ to be a good value for the star's effective temperature. About the two additional $\log g$ values, their standard deviation for iron is a bit larger than the one found in the previous section but as a whole, they are nevertheless quite similar to the ones obtained with models at $\log g$ of 6.2 . 
Table 2

$\mathrm{BD}+28^{\circ} 4211$ Chemical Composition - Results of our abundance analysis

\begin{tabular}{lcccc}
\hline \hline $\begin{array}{c}\text { Element } \\
\text { (ions) }\end{array}$ & $\begin{array}{c}\text { Nb of intervals } \\
\text { (Nb of lines) }\end{array}$ & $\begin{array}{c}\text { Mean abundance } \\
\log (\mathrm{X}) / N(\mathrm{H})\end{array}$ & $\begin{array}{c}\text { Standard deviation } \\
(\text { dex })\end{array}$ & $\begin{array}{c}\text { Total uncertainty } \\
(\text { dex })\end{array}$ \\
\hline $\mathrm{C}$ (IV) & $3(3)$ & -4.48 & 0.16 & 0.46 \\
$\mathrm{~N}$ (IV, V) & $4(8)$ & -4.23 & 0.19 & 0.78 \\
$\mathrm{O}$ (IV, V, VI) & $10(15)$ & -3.48 & 0.15 & 0.46 \\
$\mathrm{~F}$ (IV, V, VI) & $1(1)$ & -8.00 & - & 0.50 \\
$\mathrm{Mg}$ (IV) & $7(7)$ & -4.57 & 0.12 & 0.45 \\
$\mathrm{Si}$ (IV) & $3(3)$ & -4.95 & 0.06 & 0.30 \\
$\mathrm{P}$ (V) & $2(2)$ & -7.45 & 0.29 & 0.47 \\
$\mathrm{~S}$ (V, VI) & $8(8)$ & -5.53 & 0.04 & 0.43 \\
$\mathrm{Ar}$ (VI, VII) & $2(2)$ & -5.53 & 0.12 & 0.32 \\
$\mathrm{Fe}$ (V, VI, VII) & $11(33)$ & -5.08 & 0.21 & 0.48 \\
$\mathrm{Ni}$ (V, VI) & $7(17)$ & -6.04 & &
\end{tabular}

a Fluorine was not formally fitted, we visually examined some lines in order to estimate an abundance. See the text for more details.

Table 3

Chromium, manganese and cobalt estimated abundances (LTE)

\begin{tabular}{lcccc}
\hline \hline $\begin{array}{c}\text { Element } \\
\text { (ions) }\end{array}$ & $\begin{array}{c}\text { Nb of intervals } \\
\text { (Nb of lines) }\end{array}$ & $\begin{array}{c}\text { Mean abundance } \\
\log N(\mathrm{X}) / N(\mathrm{H})\end{array}$ & $\begin{array}{c}\text { Standard deviation } \\
(\text { dex })\end{array}$ & $\begin{array}{c}\text { Total uncertainty } \\
(\text { dex })\end{array}$ \\
\hline Cr (VI) & $4(4)$ & -6.17 & 0.31 & 0.41 \\
Mn (VI) & $5(6)$ & -6.71 & 0.21 & 0.32 \\
Co (VI) & $5(11)$ & -6.71 & 0.12 & 0.25 \\
\hline
\end{tabular}

We used the abundance differences between different models in the calculations of the uncertainties reported in Table 2. We wanted our chemical composition, within the given uncertainties, to be able to stand a potential change in the fundamental parameters of $\mathrm{BD}+28^{\circ} 4211$. By using the abundances found at $T_{\text {eff }}=92,000 \mathrm{~K}$ and $\log g=6.6$, our determined values should remain appropriate for changes of $\pm 10,000 \mathrm{~K}$ and $\pm 0.4 \mathrm{dex}$, assuming the errors should be symmetrical in $T_{\text {eff }}$ and $\log g$. Although we also have abundances for $72,000 \mathrm{~K}$ and $\log g$ of 5.8 we prefer not to include them in our uncertainty calculations because they are less likely to be realistic values for $\mathrm{BD}+28^{\circ} 4211$. This will be discussed in the next section. That being said, our final uncertainties are the sum of three components :

$$
\sigma=\sqrt{\left(\sigma_{\mathrm{fit}}\right)^{2}+\left(\sigma_{\mathrm{Teff}}\right)^{2}+\left(\sigma_{\mathrm{logg}}\right)^{2}}
$$

where $\sigma_{\text {fit }}$ is the standard deviation of the fitted chunks, $\sigma_{\text {Teff }}$ is the difference between the abundances at $T_{\text {eff }}=92,000 \mathrm{~K}$ and $82,000 \mathrm{~K}$ while $\sigma_{\operatorname{logg}}$ is the difference between the results at $\log g$ of 6.6 and 6.2 . For the elements besides nitrogen, silicon and iron, $\sigma_{\text {Teff }}$ and $\sigma_{\operatorname{logg}}$ were taken as the mean values of the three determined ones.

\section{CONSTRAINING THE ATMOSPHERIC PARAMETERS}

\subsection{With the Metal Lines}

As mentionned in the Introduction, finding out the effective temperatures and surface gravities of hot stars is not straightforward and standard methods used on cooler stars do not work very well. A more reliable approach in this case is to look at the metal lines visible in the UV spectrum of the star. A change of temperature will modify the ionization equilibrium of the atomic species present in the photosphere, as shown previously in Figure 2, and this will result in changes of the spectral lines strength. The exercise done in the last subsection, when fitting iron and nitrogen with models having different parameters, favors the models at $T_{\text {eff }}=82,000 \mathrm{~K}$. The standard deviations of the fitted intervals are smaller with this temperature and moreover, the ionization equilibrium of iron lines cannot be correctly reproduced neither with the hotter or cooler models. This is what is shown in Figure 6, where the two left panels feature an interval of the STIS spectrum including three ionization stages of iron and three model spectra having different temperatures. Fe VI lines do not change much with the effective temperature, but Fe $\mathrm{V}$ and VII lines are more sensitive and become quite stronger with, respectively, a lower and higher temperature. The changes are easily seen, even with a difference of $5,000 \mathrm{~K}$ between models. Thus, when trying to do a fit of the iron lines, at hotter or cooler temperatures, it is impossible to simultaneously match the lines coming from the three ionization degrees. Indeed, the four fits shown in Figure 6 (middle and right panels) are rather poor. Therefore, when looking at iron lines as a temperature indicator, it appears clearly that the temperature of the star must be quite close to 82,000 K.

Figure 7 shows the same kind of plots than Figure 6, except that this time we changed the value of the surface gravity between $\log g$ of 6.6 and 5.8 while the temperature was fixed at $T_{\text {eff }}=82,000 \mathrm{~K}$. Like the temperature, the gravity also affects the iron lines, but to a lesser extend, at least in the ranges investigated. With a higher $\log g$ it is still possible to represent correctly the lines shown in the two right panels, but the fits lead to abundances that are different by 0.45 dex, which is larger than the difference of 0.12 dex obtained with our models at log 
Table 4

Determined abundances of nitrogen, silicon and iron

\begin{tabular}{lcccccc}
\hline \hline Models & \multicolumn{2}{c}{ Nitrogen } & \multicolumn{2}{c}{ Silicon } & \multicolumn{2}{c}{ Iron } \\
& Abundance & $\sigma$ & Abundance & $\sigma$ & Abundance & $\sigma$ \\
\hline$T_{\text {eff }} 82 \mathrm{kK}$ & -4.23 & 0.19 & -4.95 & 0.06 & -5.07 & 0.12 \\
$T_{\text {eff }} 92 \mathrm{kK}$ & -3.47 & 0.32 & -4.66 & 0.05 & -4.83 & 0.58 \\
$T_{\text {eff } 72 \mathrm{kK}}$ & -4.64 & 0.33 & -5.32 & 0.13 & $-5.03^{\mathrm{a}}$ & $0.35^{\mathrm{a}}$ \\
$\log g 6.6$ & -4.24 & 0.16 & -4.95 & 0.02 & -4.89 & 0.20 \\
$\log g 5.8$ & -4.15 & 0.18 & -4.94 & 0.13 & -5.23 & 0.14 \\
& & & & & & \\
\hline
\end{tabular}

a The quality of the iron fits with these models is rather poor.
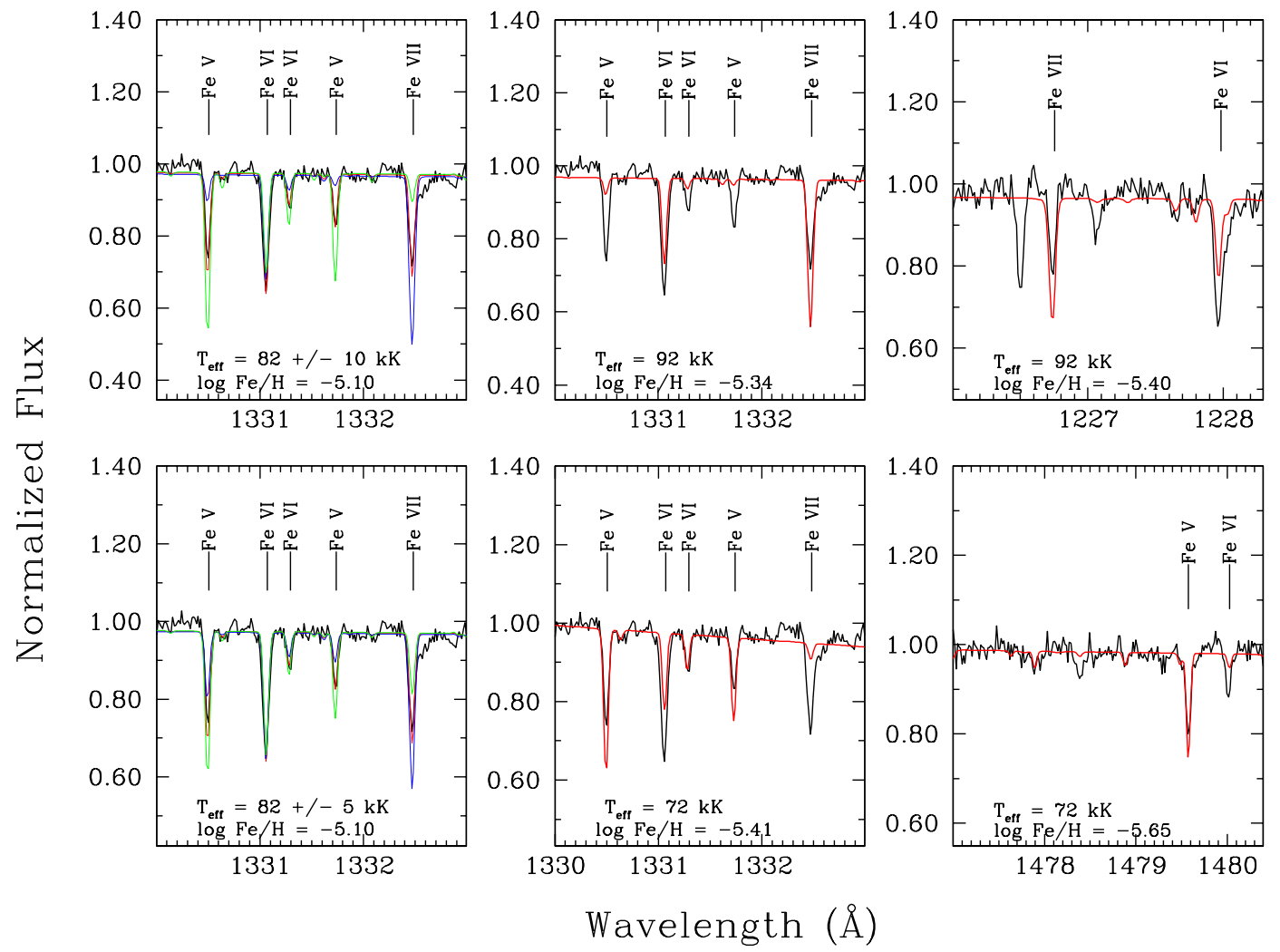

Figure 6. Comparison of iron lines with models having different temperatures. The temperature and the abundance of iron are indicated on each panel. The two left panels present a comparison of three model spectra with the spectrum of $\mathrm{BD}+28^{\circ} 4211$ over a range featuring lines from three ionization stages of iron. The standard model at $T_{\text {eff }}=82,000 \mathrm{~K}$ and $\log g=6.2$ is represented by the red line, the hotter ones $(92,000 \mathrm{~K}$ in the top panel and $87,000 \mathrm{~K}$ in the bottom one) are in blue, while the cooler models $(72,000 \mathrm{~K}$ in the top panel and 77,000 $\mathrm{K}$ in the bottom one) are in green. It can be seen that with a fixed abundance, a change in the temperature, even of only $5,000 \mathrm{~K}$, leads to a poorer agreement between the observed lines and the modeled ones. The two middle panels show the result of a fitting procedure, over the same wavelength range, with hotter and cooler models, while the right panels also show a fit of iron lines, but over two other spectral ranges.

$g=6.2$ (see the online Figure, panel g). When fitting our principal range of interest, $1330-1333 \AA$, with models having different $\log g$, we do not obtain a match as good as with our reference grid. Indeed, when looking at the standard deviations for the iron fits found in Table 4 for the various $\log g$, the agreement is better with the reference model, but $\sigma$ is not much higher in the two other cases. In the case of nitrogen, the standard deviations of the various $\log g$ do not obviously support a specific gravity. That being said, when considering iron lines, the surface gravity we assumed for our analyses $(\log g=6.2)$ seems to be right.

\subsection{With the Parallax Distance}

Another method that can be used to place constraints on the parameters of $\mathrm{BD}+28^{\circ} 4211$ is to compare its spectroscopic distance with the one determined by parallax measurements, which is between 81 and 106 pc according to the latest reduction of the Hipparcos catalogue 

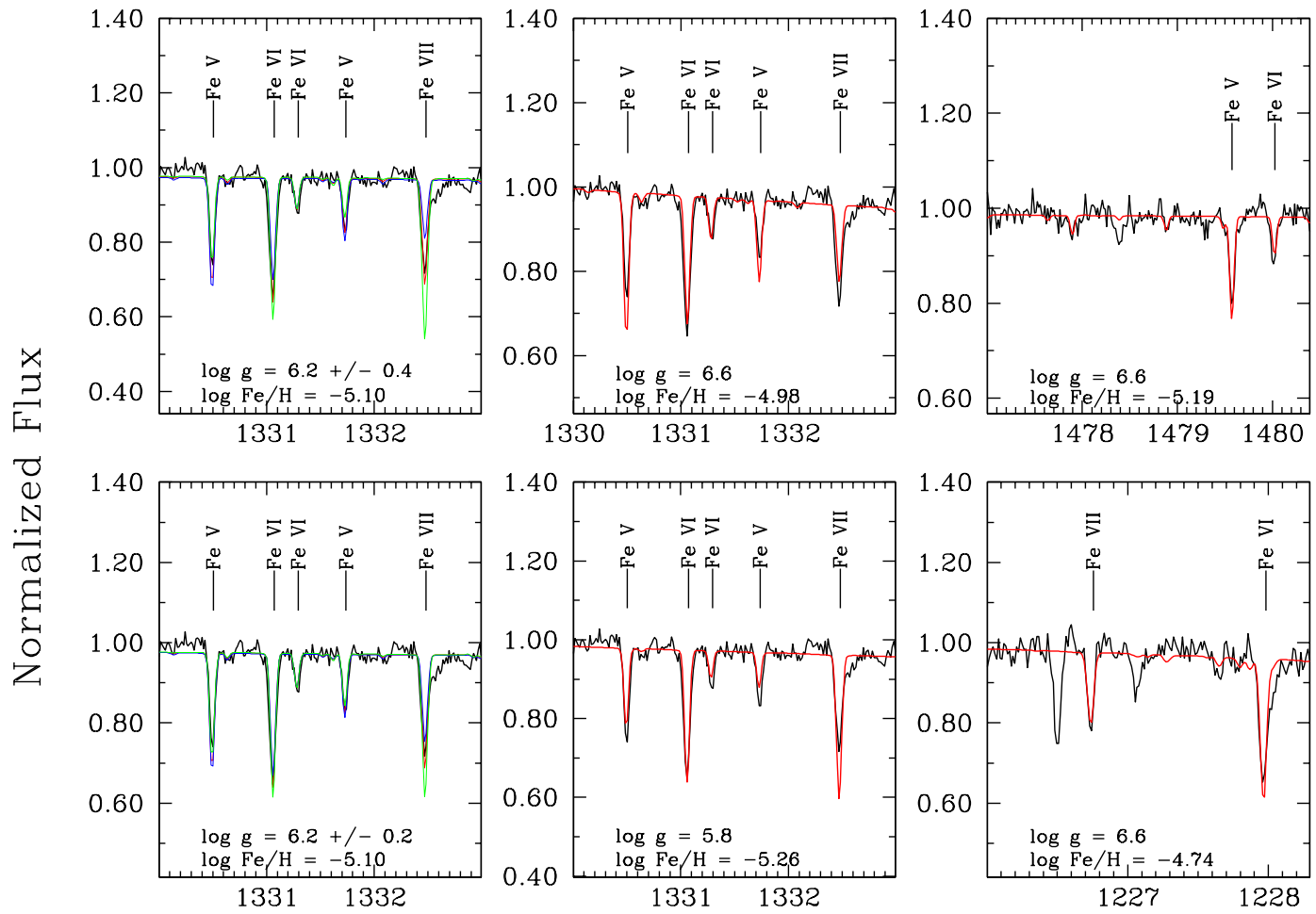

Wavelength $(\AA)$

Figure 7. Comparisons of iron lines with models at different $\log g$. This figure is very similar to Figure 6 , but for different values of log $g$ while the temperature is kept at $82,000 \mathrm{~K}$. The two left panels show the observed spectrum and three models with different surface gravity, having a fixed iron abundance. Models in the upper panel have gravities that change by 0.4 dex with $\log g=6.2$ as a middle value, while the changes are of $0.2 \mathrm{dex}$ in the lower panel. The spectrum with the highest value is in blue, the middle one in red, and the lowest one in green. The two middle panels show the results of the fits with the two extreme values of $\log g$, while the right panels show different ranges, fitted with models at $\log g$ of 6.6 .

(van Leeuwen 2007). The idea here is to compute the absolute magnitude of a model atmosphere and combine it with the apparent magnitude of the star (recently mesured by Landolt \& Uomoto (2007)) to get the distance. In order to do that, some other quantities are required.

First of all, at these distances, reddening must be considered, and we thus computed theoretical, unreddened $(B-V)_{o}$ color indices for several relevant model spectra. We used the flux calibration of Holberg \& Bergeron (2006) to find the absolute $B$ and $V$ magnitudes. By considering six models (with $T_{\text {eff }}=82,000 \mathrm{~K}$ and 87,000 $\mathrm{K}$, and $\log g=6.2,6.4$, and 6.6 ), we derived an average representative color index $(B-V)_{o}=-0.3831 \pm 0.0018$ showing a very small dispersion (which is, of course, not surprising for a star as hot as BD+28 4211 ). This is to be compared with the accurate observed value of $(B-$ $V)=-0.3410 \pm 0.0018$ obtained bynLandolt \& Uomoto (2007), leading immediately to a rather precise reddening index of $E(B-V)=0.042 \pm 0.003$ for $\mathrm{BD}+28^{\circ} 4211$. Combined with the extinction law proposed by Seaton (1979), this leads to an absorption coefficient in the $V$ band of $A_{V}=3.20 E(B-V)=0.135 \pm 0.008$. We note that this value is quite compatible with the overall ab- sorption coefficient of $A_{V}=0.3$ along the line-of-sight in the direction of $\mathrm{BD}+28^{\circ} 4211$ as obtained from the Infrared Science Archive ${ }^{7}$ using data from Schlegel et al. (1998).

Another parameter needed in the computation of the spectroscopic distance is the mass of the star, which allows to find its radius given the surface gravity. Unfortunately, that parameter is largely unknown, except to say that $\mathrm{BD}+28^{\circ} 4211$ must be either a post-AGB, a post$\mathrm{EHB}$, or maybe a post-RGB (though this is less likely because of the relatively short timescale of this evolutionary path). Constraints on the mass can then be derived from model calculations of these late evolutionary phases. For instance, according to the evolutionary tracks of Schoenberner (1983) (post-AGB), Dorman et al. (1993) (post-EHB), and Driebe et al. (1998) (post-RGB) and the approximate position of $\mathrm{BD}+28^{\circ} 4211$ in the $\log g$ $-T_{\text {eff }}$ plane, it would appear that its mass should be around $0.5 M_{\odot}$ and could hardly be higher than 0.6 $M_{\odot}$ or lower than $0.4 M_{\odot}$. Plots of post-AGB and post-EHB tracks can be found in Figure 1 of Haas et al. (1996), while post-RGB tracks are shown in Figure 10 of

\footnotetext{
${ }^{7}$ http://irsa.ipac.caltech.edu/applications/DUST/
} 
Table 5

Spectroscopic distances (pc) obtained with models having $T_{\text {eff }}=82,000 \mathrm{~K}$ in $V$-band with $\mathrm{A}_{\mathrm{v}}=0$ and 0.135

\begin{tabular}{ccccc}
\hline \hline Mass $/ \log g$ & $0.3 M_{\odot}$ & $0.4 M_{\odot}$ & $0.5 M_{\odot}$ & $0.6 M_{\odot}$ \\
\hline $\log g=6.2$ & $129 / 122$ & $150 / 140$ & $167 / 157$ & $183 / 172$ \\
$\log g=6.4$ & $103 / 97$ & $119 / 111$ & $133 / 125$ & $145 / 136$ \\
$\log g=6.6$ & $81 / 77$ & $94 / 88$ & $105 / 99$ & $115 / 108$ \\
\hline
\end{tabular}

Stroeer et al. (2007). However, this is probably not the full story because we know of post-EHB stars with masses less than $0.4 M_{\odot}$, one of which not known to be part of a close binary system (Heber et al. (2005); Randall et al. (2007); For et al. (2010); Fontaine et al. (2012)). We cannot therefore exclude a mass for $\mathrm{BD}+28^{\circ} 4211$ less than $0.4 M_{\odot}$ and, as an extreme limit, we will also consider a value as low as $0.3 M_{\odot}$.

Finally, the surface gravity and the effective temperature of a given model atmosphere influence direcly the absolute magnitude determined in a given bandpass and, thus, the inferred distance. Since the effective temperature of $\mathrm{BD}+28^{\circ} 4211$ appears to be well constrained by the pattern of iron lines, this parameter was initially fixed at $T_{\text {eff }}=82,000 \mathrm{~K}$ and we computed the spectroscopic distance of the star for different combinations of mass and surface gravity. This was done by comparing the computed absolute visual magnitude $M_{V}$ with the well-measured reddened apparent magnitude of $V=10.509 \pm 0.0027$ provided by Landolt \& Uomoto (2007)

Our results are presented in Table 5, where, for each combination of mass and gravity, we computed two distances, with and without the reddening. The comparison of our computed spectroscopic distances and the measured one favors a surface gravity higher than 6.2 and/or a low mass for $\mathrm{BD}+28^{\circ} 4211$. If we were to insist that the mass of $\mathrm{BD}+28^{\circ} 4211$ is a representative post-EHB star value, $0.5 M_{\odot}$ say, then our optimal spectroscopic model - characterized by $T_{\text {eff }}=82,000 \mathrm{~K}$ and $\log g=$ 6.2 - would lead to a distance of $157 \mathrm{pc}$, in apparent conflict with the parallax measurement of $81-106 \mathrm{pc}$. This is very reminescent of the situation encountered by Rauch et al. (2007) in the case of the hot DAO white dwarf LS V $+46^{\circ} 21$ where the authors estimated the unknown mass by interpolating in a given set of evolutionary tracks. With a fixed value of $0.55 M_{\odot}$, they found a discrepant spectrocopic distance of $224_{-58}^{+46}$ pc compared to a ground-based parallax measurement giving $129_{-5}^{+6}$ pc.

If we take the parallax measurement of $\mathrm{BD}+28^{\circ} 4211$ at face value, then the surface gravity has to be pushed above $\log g \sim 6.5$ for our spectroscopic distance to become compatible with that measurement, again assuming that the mass of the star is $0.5 M_{\odot}$. However, such large values of the surface gravity are now in conflict with the iron line profiles depicted in Figure 7. It may thus be preferable to think in terms of a low mass for $\mathrm{BD}+28^{\circ} 4211$ for the time being. This may also be an option in the case of LS V $+46^{\circ} 21$ (Rauch et al. 2007).

Finally, we also checked what would be the effect of a change in the temperature of our model spectra and we thus computed a table similar to Table 5, except that all models were characterized by $T_{\text {eff }}=87,000 \mathrm{~K}$ instead of $82,000 \mathrm{~K}$. We found that the spectroscopic distance increases by only 2 to $6 \mathrm{pc}$, depending on the parameters mass and gravity, compared to the entries of Table 5. Hence, within an uncertainty of $5000 \mathrm{~K}$ (which seems to be a reasonable range according to the result of the previous section), the conclusions of this subsection are practically not dependent on the effective temperature.

\section{DISCUSSION}

In spite of the huge progress made in the model atmosphere modeling field in the last two decades, the analysis of hot stars still remains a challenge. In this paper, we have presented the first part of our analysis of $\mathrm{BD}+28^{\circ} 4211$, which consists in the study of its UV spectrum. Our work made use of high-quality spectra from the $H S T$ and FUSE satellites combined with stateof-the-art NLTE line-blanketed model atmospheres and synthetic spectra computed with TLUSTY and SYNSPEC. To our knowledge, the FUSE data available on that star have not been exploited previously in the context of an atmospheric abundance analysis. The abundances of eleven elements have been determined, namely those of C, N, O, F, Mg, Si, P, S, Ar, Fe, and Ni. Our abundance analysis was made in a self-consistent way, meaning that the element analyzed contributed to the thermodynamical structure of the model and its populations were explicitly calculated in NLTE during the computation of the model atmosphere. We also made sure that the models used for fitting elements always included, besides the fitted element, at least the atomic species that most contribute to the thermodynamical structure, that is to say helium, carbon, nitrogen, oxygen, and iron. For a star as hot as BD+28 4211 , we stress that it is crucial to compute NLTE populations of the studied elements in order to get realistic abundances. Even with the thermodynamical structure of a NLTE line-blanketed model atmosphere, the LTE ionization equilibrium is likely to be wrong, thus preventing a simultaneous fit of absorption lines originating from different ionization stages of a given atomic species. We illustrated this effect in Figure 3, with an example of nickel lines computed in both the NLTE self-consistent way and with LTE populations. In addition to the elements mentionned above, the UV spectrum of $\mathrm{BD}+28^{\circ} 4211$ shows lines of chromium, manganese, and cobalt (and probably those of other species as well). We tentatively tried to fit these three elements by using the LTE approximation, because of the lack of proper model atoms that could be used in our NLTE models. We analyzed only lines originating from the dominant ionization stage (VI) and we got abundances surprisingly similar to the ones Ramspeck et al. (2003) derived (for $\mathrm{Cr}$ and $\mathrm{Mn}$ ) using the "generic ion" approach in NLTE model atmosphere.

Our resulting chemical composition is summed up in Figure 8 and the entire sample of our NLTE fitted lines is shown in the online Figure. We found the overall quality of our fits very satisfying. When comparing the abundances of $\mathrm{BD}+28^{\circ} 4211$ with those of the Sun, it appears that none of the elements studied with self-consistent NLTE models has an abundance higher than solar (the only exceptions being the LTE estimated abundances of chromium and cobalt). Instead, our derived abundances are all between one and 1/10 of the solar ones 
(Asplund et al. 2009). The most depleted species is carbon with $\log N(\mathrm{C}) / N\left(\mathrm{C}_{\odot}\right)=-0.91 \pm 0.46$, followed by phophorus with $\log N(\mathrm{P}) / N\left(\mathrm{P}_{\odot}\right)=-0.86 \pm 0.52$. It turns out that these two elements, as mentionned in subsection 2.2, are present in the photosphere mainly as $\mathrm{C}$ V and $\mathrm{P}$ VI, which are in a noble gas configuration. This kind of ion usually has its resonance lines in the extreme UV (EUV) or even in the X-ray domain because of the large energy gap between their ground and first excited levels. Therefore, they are less sensitive to radiative levitation, since the EUV and X-ray fluxes of stars are usually lower than the flux at larger wavelengths. This might explain why carbon and phosphorus are the most depleted elements, since their resonance lines are respectively around $40 \AA$ and $90 \AA$. Even if BD $+28^{\circ} 4211$ is a hot star, its flux at these extreme wavelengths remains quite low. For its part, even though our abundance of fluorine is at best an estimation based on a sole line, its value around $\log N(\mathrm{~F}) / N\left(\mathrm{~F}_{\odot}\right)=-0.56 \pm 0.50$ still suggests that this element is not enriched in $\mathrm{BD}+28^{\circ} 4211$. This is in line with the findings of Werner et al. (2005) who found an important enrichment of fluorine in a number of PG1159 stars, while its abundance remains around or slighly lower than solar in their sample of H-rich central stars of planetary nebulae, the latter being a family of stars having similar evolutionary paths than $\mathrm{BD}+28^{\circ} 4211$. As for iron, our results indicate that $\log N(\mathrm{Fe}) / N\left(\mathrm{Fe}_{\odot}\right)=-0.58 \pm 0.32$, while they indicate $\log N(\mathrm{Ni}) / N\left(\mathrm{Ni}_{\odot}\right)=-0.26 \pm 0.48$ for nickel. Both results are compatible with the findings of Ramspeck et al. (2003) which suggest a depleted value of iron by about an order of magnitude (formally, from their Table 1, log $\left.N(\mathrm{Fe}) / N\left(\mathrm{Fe}_{\odot}\right)=-0.82 \pm 0.15\right)$, and a solar abundance for nickel $^{8}$. We thus find a ratio $N(\mathrm{Fe}) / N(\mathrm{Ni}) \simeq 9.1$,

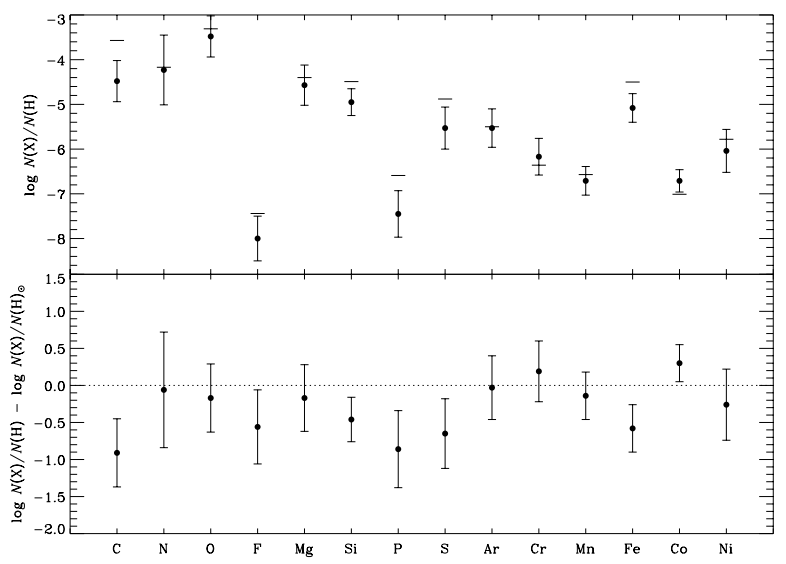

Figure 8. Summary of the determined chemical composition of $\mathrm{BD}+28^{\circ} 4211$. The top panel shows the absolute abundances relative to hydrogen $(\log N(\mathrm{X}) / N(\mathrm{H}))$ and the horizontal lines indicate the solar one for each element (Asplund et al. 2009). The bottom panel shows this time the abundances relative to the solar value.

${ }^{8}$ Our derived abundances $\log N(\mathrm{~N}) / N\left(\mathrm{~N}_{\odot}\right)=-0.06 \pm 0.78$ and $\log N(\mathrm{O}) / N\left(\mathrm{O}_{\odot}\right)=-0.17 \pm 0.46$ are equally compatible with the work of Haas et al. (1996) who deduced about solar abundances which is about half of the solar value. An even more depleted ratio was first suggested by Haas et al. (1996) in $\mathrm{BD}+28^{\circ} 4211$ and is particularly interesting because it connects well with the work of Werner \& Dreizler (1994) who found a similar trend in four hot DA white dwarfs. This trend may possibly be explained through the combined effects of radiative levitation and residual stellar winds (see, e.g., the work of Chayer et al. (1994)). Both elements have similar atomic masses and structures, but the lower ratios obtained in hot stars suggest that nickel is more sensitive to radiative levitation and/or winds than iron, thus allowing a larger proportion of nickel to stay in the photosphere. It must be kept in mind that with an effective temperature of $82,000 \mathrm{~K}$, the abundance pattern of $\mathrm{BD}+28^{\circ} 4211$ is strongly affected by radiative levitation. We made sure that the uncertainties on our determined abundances were computed in a way that includes the effects of a change in the temperature and the gravity of our model atmospheres. Therefore, our abundances should remain fairly reliable even if the effective temperature or the surface gravity are revised upward or downward by some $10,000 \mathrm{~K}$ or 0.4 dex, respectively ${ }^{9}$.

Even if we took the fundamental parameters of $\mathrm{BD}+28^{\circ} 4211$ from the literature (Napiwotzki 1993), we nevertheless checked the validity of the assumed effective temperature and surface gravity. To carry out that task, we first redid our fits for nitrogen, silicon, and iron (used as proxy elements) by constructing additional model atmospheres having different parameters. The standard deviation of the sample of fitted intervals for an element gives us an idea of how well the different abundances agree internally with each other. A small value of $\sigma$ indicates that all intervals lead to similar abundances, which is a good thing in our case. By examining the standard deviations obtained when the temperature of the models is changed, we found the results to be in better agreement when the effective temperature of our models is near 82,000 K, which is the assumed one. As for the standard deviations obtained with various $\log g$ values, they do not point toward a favored value of the surface gravity. Instead, we have to look at a comparison of observed and synthetic spectra over a wavelength range featuring iron lines originating from three ionization stages, namely Fe V, VI, and VII to have an indication of the better value for the surface gravity (see Figure 7). This figure suggests the assumed value of 6.2 dex to be the one giving the best simultaneous match of the iron lines over the selected range. The differences between our model spectra at different $\log g$ are not as striking as the one we obtained with different $T_{\text {eff }}$ (see Figure 6 ), but they are nevertheless significant.

We also exploited the availability of an Hipparcos parallax measurement for $\mathrm{BD}+28^{\circ} 4211$ and compared the inferred distance with spectroscopic distances estimated from several model spectra. In a first step, we were able to derive an accurate determination of the reddening between Earth and $\mathrm{BD}+28^{\circ} 4211, E(B-V)=$ $0.042 \pm 0.003$, thanks to the high-precision optical photometry of Landolt \& Uomoto (2007). Several spectro-

for those two elements.

9 After our complete analysis, it appears that a $\pm 10,000 \mathrm{~K}$ margin of error on the effective temperature is twice our adopted value. Thus, the uncertainties on our abundances are a bit generous. 
scopic distances (with and without reddening correction) were derived as indicated in Table 5. A comparison with the parallax distance implies a relatively large value of the surface gravity and/or a small mass for $\mathrm{BD}+28^{\circ} 4211$. We can reconcile our spectroscopic constraints with the available parallax measurement only if the mass of $\mathrm{BD}+28^{\circ} 4211$ is significantly less than the canonical value of $0.5 M_{\odot}$ for a representative post-EHB star. Assuming that the Hipparcos measurement for $\mathrm{BD}+28^{\circ} 4211$ is fully reliable and that our model atmospheres are reasonably realistic, we must conclude that $\mathrm{BD}+28^{\circ} 4211$ is likely less massive than could have been expected on the basis of standard evolutionary tracks.

Our analysis has allowed us to get a good idea of the atmospheric chemical composition of $\mathrm{BD}+28^{\circ} 4211$. Its main constituents, in terms of atomic species, have been analyzed and we were able to get abundances for eleven elements, including the ones that influence the most the thermodynamical structure, i.e., carbon, nitrogen, oxygen, and iron. This now allows us to compute more realistic NLTE model atmospheres including the appropriate line blanketing for $\mathrm{BD}+28^{\circ} 4211$. The value of $82,000 \mathrm{~K}$ for the effective temperature of the star now seems quite robust and realistic uncertainties are likely less than $\pm 5000 \mathrm{~K}$. The case of the surface gravity is somewhat more difficult, but we estimate conservatively that $\log g=6.2_{-0.1}^{+0.3}$ for $\mathrm{BD}+28^{\circ} 4211$. These informations should be a good starting point for the study of the optical spectrum of the star. This will follow in an upcoming paper.

This work was supported in part by the NSERC of Canada. G.F. also acknowledges the contribution of the Canada Research Chair Program. M.L. wishes to thank Patrick Dufour for help with the fitting routines.

\section{REFERENCES}

Asplund, M., Grevesse, N., Sauval, A. J., \& Scott, P. 2009, ARA\&A, 47, 481

Ayres, T. R. 2010, ApJS, 187, 149

Bergeron, P., Saffer, R. A., \& Liebert, J. 1992, ApJ, 394, 228

Bruhweiler, F. C. \& Dean, C. A. 1983, ApJ, 274, L87

Chayer, P., Fontaine, M., Fontaine, G., Wesemael, F., \& Dupuis, J. 2006, Baltic Astronomy, 15, 131

Chayer, P., LeBlanc, F., Fontaine, G., Wesemael, F., \& Vennes, S. 1994, ApJ, 436, L161

Chayer, P., Vennes, S., Dupuis, J., \& Kruk, J. W. 2005, ApJ, 630, L169

Dixon, W. V., Sahnow, D. J., Barrett, P. E., Civeit, T., Dupuis, J., Fullerton, A. W., Godard, B., Hsu, J.-C., Kaiser, M. E., Kruk, J. W., Lacour, S., Lindler, D. J., Massa, D., Robinson, R. D., Romelfanger, M. L., \& Sonnentrucker, P. 2007, PASP, 119,527

Dorman, B., Rood, R. T., \& O'Connell, R. W. 1993, ApJ, 419, 596

Dreizler, S. \& Werner, K. 1993, A\&A, 278, 199

Driebe, T., Schoenberner, D., Bloecker, T., \& Herwig, F. 1998, A\&A, 339, 123

Fontaine, G., Brassard, P., Charpinet, S., \& et al. 2012, A\&A, 539, A12

For, B.-Q., Green, E. M., Fontaine, G., \& et al. 2010, ApJ, 708, 253

Grevesse, N. \& Sauval, A. J. 1998, Space Sci. Rev., 85, 161

Haas, S., Dreizler, S., Heber, U., Jeffery, S., \& Werner, K. 1996 , A\&A, 311, 669

Heber, U., Drechsel, H., Karl, C., Østensen, R., B., \& Koester, D. 2005, in Astronomical Society of the Pacific Conference Series, Vol. 334, 14th European Workshop on White Dwarfs, ed. D. Koester \& S. Moehler, 357
Herbig, G. H. 1999, PASP, 111, 1144

Holberg, J. B. \& Bergeron, P. 2006, AJ, 132, 1221

Landolt, A. U. \& Uomoto, A. K. 2007, AJ, 133, 768

Lanz, T. \& Hubeny, I. 2003, ApJS, 146, 417

-. 2007, ApJS, 169, 83

Lanz, T., Hubeny, I., \& de Koter, A. 1996, Physica Scripta

Volume T, 65, 144

Latour, M., Fontaine, G., Brassard, P., Green, E. M., Chayer, P., \& Randall, S. K. 2011, ApJ, 733, 100

Massey, P. \& Gronwall, C. 1990, ApJ, 358, 344

Moos, H. W., Cash, W. C., Cowie, L. L., Davidsen, A. F., Dupree, A. K., Feldman, P. D., Friedman, S. D., Green, J. C., Green, R. F., Gry, C., Hutchings, J. B., Jenkins, E. B., Linsky, J. L., Malina, R. F., Michalitsianos, A. G., Savage, B. D., Shull, J. M., Siegmund, O. H. W., Snow, T. P., Sonneborn, G., Vidal-Madjar, A., Willis, A. J., Woodgate, B. E., York, D. G., Ake, T. B., Andersson, B.-G., Andrews, J. P., Barkhouser, R. H., Bianchi, L., Blair, W. P., Brownsberger, K. R., Cha, A. N., Chayer, P., Conard, S. J., Fullerton, A. W., Gaines, G. A., Grange, R., Gummin, M. A., Hebrard, G., Kriss, G. A., Kruk, J. W., Mark, D., McCarthy, D. K., Morbey, C. L., Murowinski, R., Murphy, E. M., Oegerle, W. R., Ohl, R. G., Oliveira, C., Osterman, S. N., Sahnow, D. J., Saisse, M., Sembach, K. R., Weaver, H. A., Welsh, B. Y., Wilkinson, E., \& Zheng, W. 2000, ApJ, 538, L1

Napiwotzki, R. 1993, Acta Astronomica, 43, 343

- 1999, A\&A, 350, 101

O'Toole, S. J. \& Heber, U. 2006, A\&A, 452, 579

Ramspeck, M., Haas, S., Napiwotzki, R., Heber, U., Deetjen, J., \& Dreizler, S. 2003, in Astronomical Society of the Pacific Conference Series, Vol. 288, Stellar Atmosphere Modeling, ed. I. Hubeny, D. Mihalas, \& K. Werner, 161

Randall, S. K., Green, E. M., Van Grootel, V., Fontaine, G., Charpinet, S., Lesser, M., Brassard, P., Sugimoto, T., Chayer, P., Fay, A., Wroblewski, P., Daniel, M., Story, S., \& Fitzgerald, T. 2007, A\&A, 476, 1317

Rauch, T., Ziegler, M., Werner, K., Kruk, J. W., Oliveira, C. M., Vande Putte, D., Mignani, R. P., \& Kerber, F. 2007, A\&A, 470,317

Saffer, R. A., Bergeron, P., Koester, D., \& Liebert, J. 1994, ApJ, 432,351

Sahnow, D. J., Moos, H. W., Ake, T. B., Andersen, J.,

Andersson, B.-G., Andre, M., Artis, D., Berman, A. F., Blair, W. P., Brownsberger, K. R., Calvani, H. M., Chayer, P., Conard, S. J., Feldman, P. D., Friedman, S. D., Fullerton, A. W., Gaines, G. A., Gawne, W. C., Green, J. C., Gummin, M. A., Jennings, T. B., Joyce, J. B., Kaiser, M. E., Kruk, J. W., Lindler, D. J., Massa, D., Murphy, E. M., Oegerle, W. R., Ohl, R. G., Roberts, B. A., Romelfanger, M. L., Roth, K. C., Sankrit, R., Sembach, K. R., Shelton, R. L., Siegmund, O. H. W., Silva, C. J., Sonneborn, G., Vaclavik, S. R., Weaver, H. A., \& Wilkinson, E. 2000, ApJ, 538, L7

Schlegel, D. J., Finkbeiner, D. P., \& Davis, M. 1998, ApJ, 500, 525

Schoenberner, D. 1983, ApJ, 272, 708

Seaton, M. J. 1979, MNRAS, 187, 73P

Sonneborn, G., André, M., Oliveira, C., Hébrard, G., Howk, J. C., Tripp, T. M., Chayer, P., Friedman, S. D., Kruk, J. W., Jenkins, E. B., Lemoine, M., Moos, H. W., Oegerle, W. R., Sembach, K. R., \& Vidal-Madjar, A. 2002, ApJS, 140, 51

Stroeer, A., Heber, U., Lisker, T., Napiwotzki, R., Dreizler, S., Christlieb, N., \& Reimers, D. 2007, A\&A, 462, 269

van Leeuwen, F. 2007, A\&A, 474, 653

Vennes, S., Chayer, P., \& Dupuis, J. 2005, ApJ, 622, L121

Wassermann, D., Werner, K., Rauch, T., \& Kruk, J. W. 2010, A\&A, 524, A9

Werner, K. 1996, ApJ, 457, L39

Werner, K. \& Dreizler, S. 1994, A\&A, 286, L31

Werner, K., Heber, U., \& Fleming, T. 1994, A\&A, 284, 907

Werner, K., Rauch, T., \& Kruk, J. W. 2005, A\&A, 433, 641

Werner, K., Rauch, T., Ringat, E., \& Kruk, J. W. 2012, ApJ, $753, \mathrm{~L} 7$ 


\section{ONLINE FIGURES}

Online Figure - The online figure present the totality of our fitted spectral intervals for all the atomic species. The fits were done with models having $T_{\text {eff }}=82,000 \mathrm{~K}$, $\log g=6.2$, and $\log N(\mathrm{He}) / N(\mathrm{H})=-1.0$. The red curve shows the result of the fitting procedure for the element mentionned on each panel and the abundance obtained is expressed as $\log N(\mathrm{X}) / N(\mathrm{H})$. a) The fit showed in the lower left panel was not included in our mean abundance for nitrogen. c) The O IV line at $1338.6 \AA$ is fitted twice because each line comes from two different STIS orders. They were both included in the mean abundance. The lower right panel shows a view of He II $\lambda 1640$, with the oxygen abundance fixed to its determined value. g) In the middle top panel, the line at the left is Co vi $\lambda 1226.4$, an element not included in our model atmospheres. 


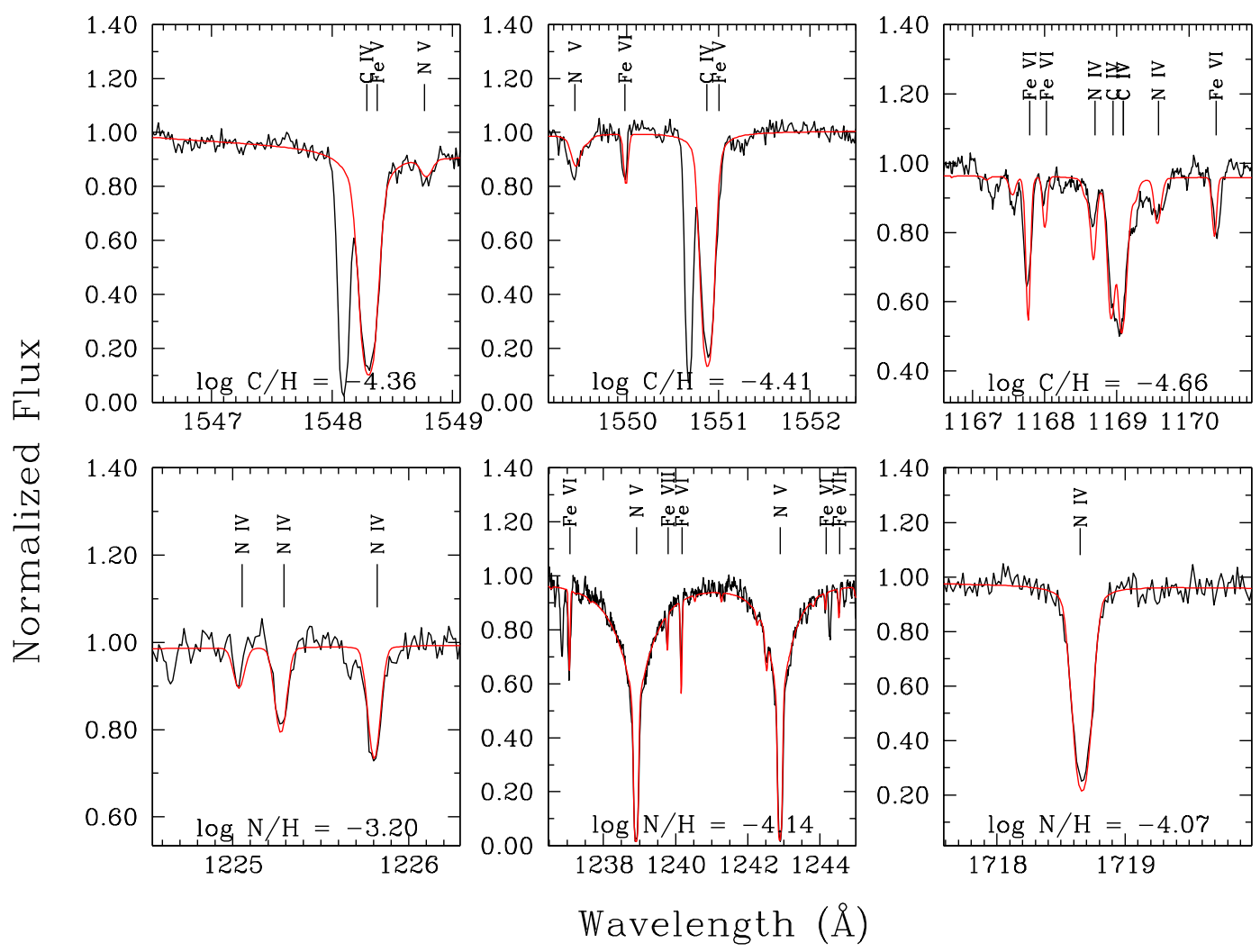

Online Figure panel a

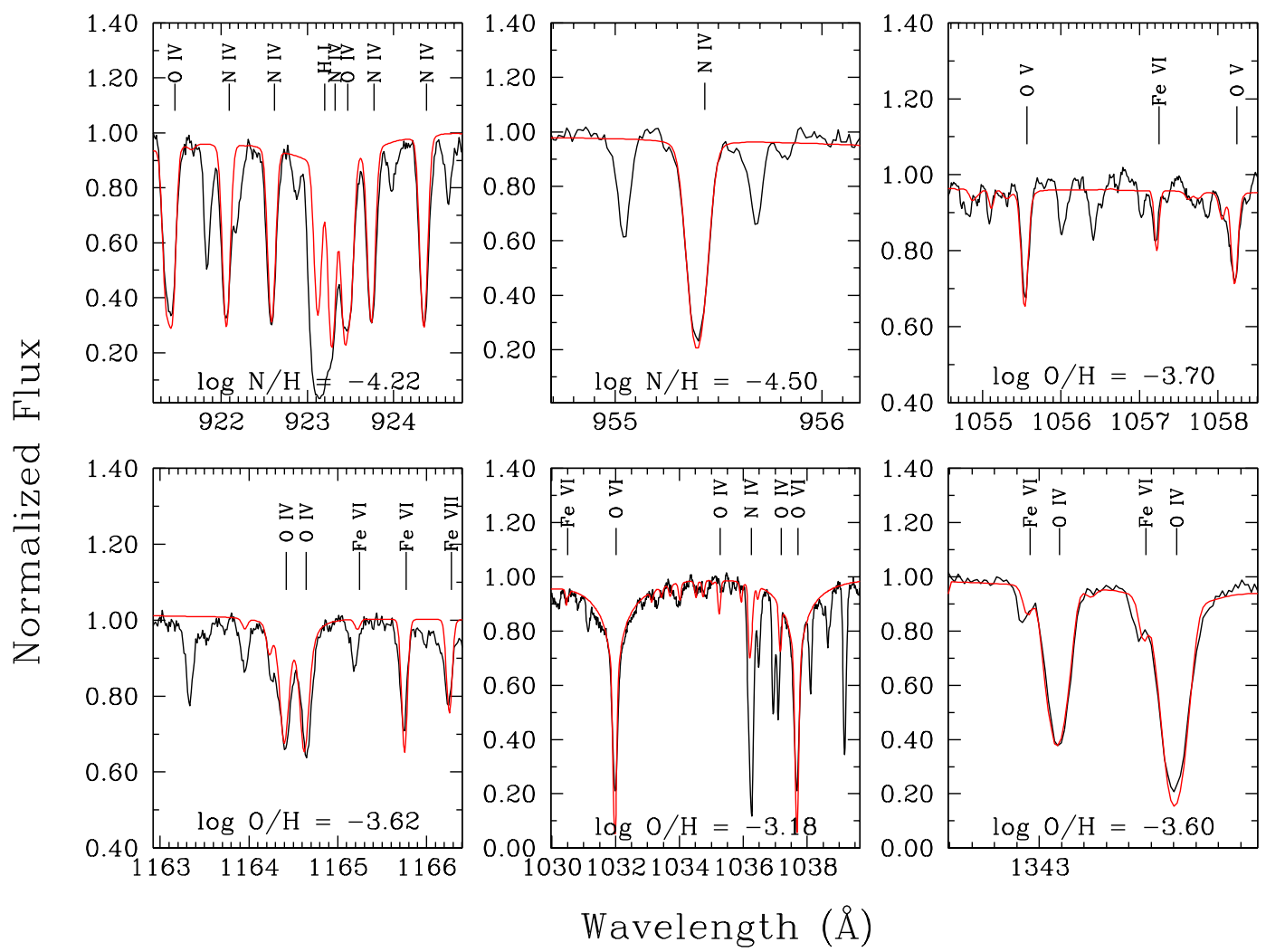

Online Figure panel b 


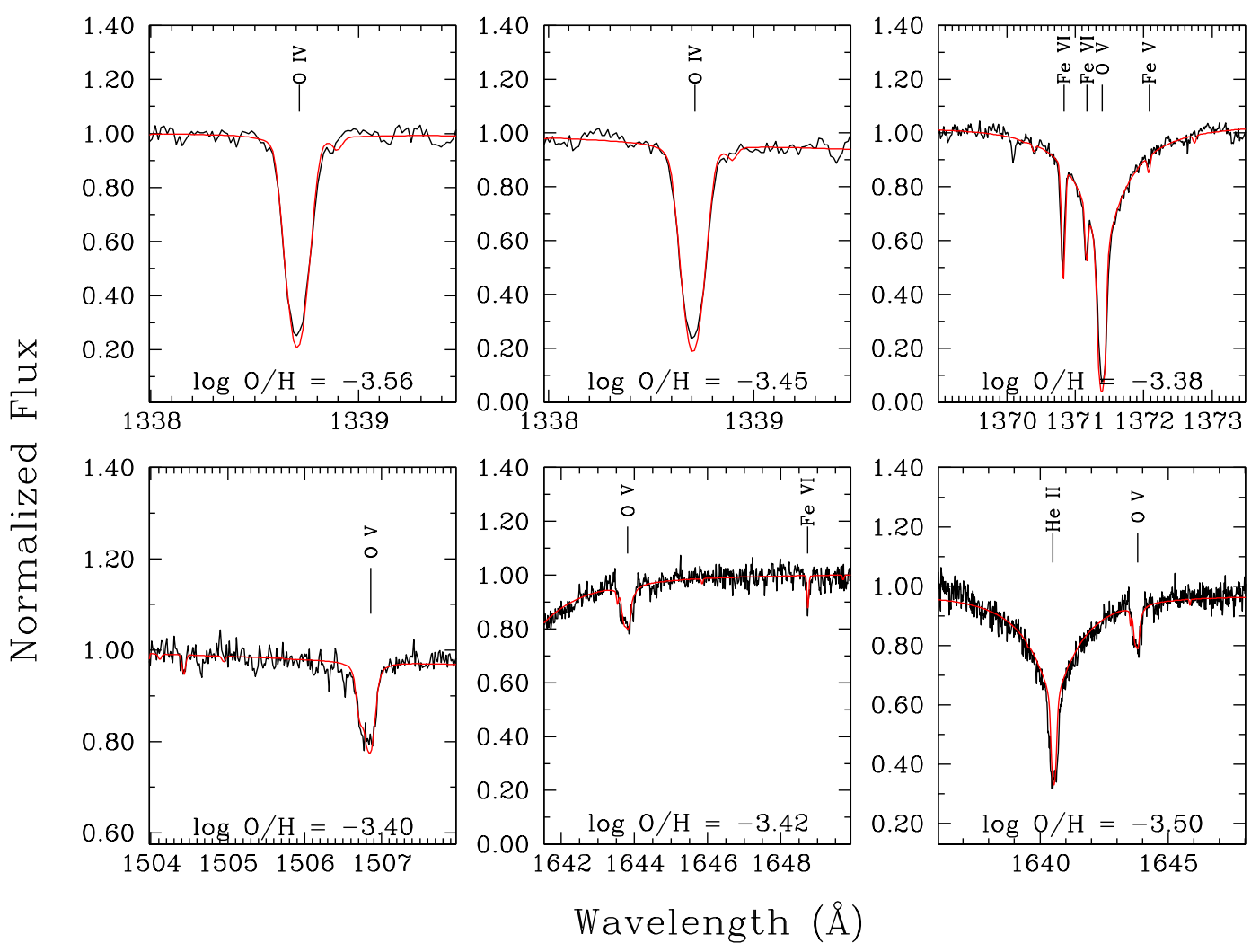

Online Figure panel c
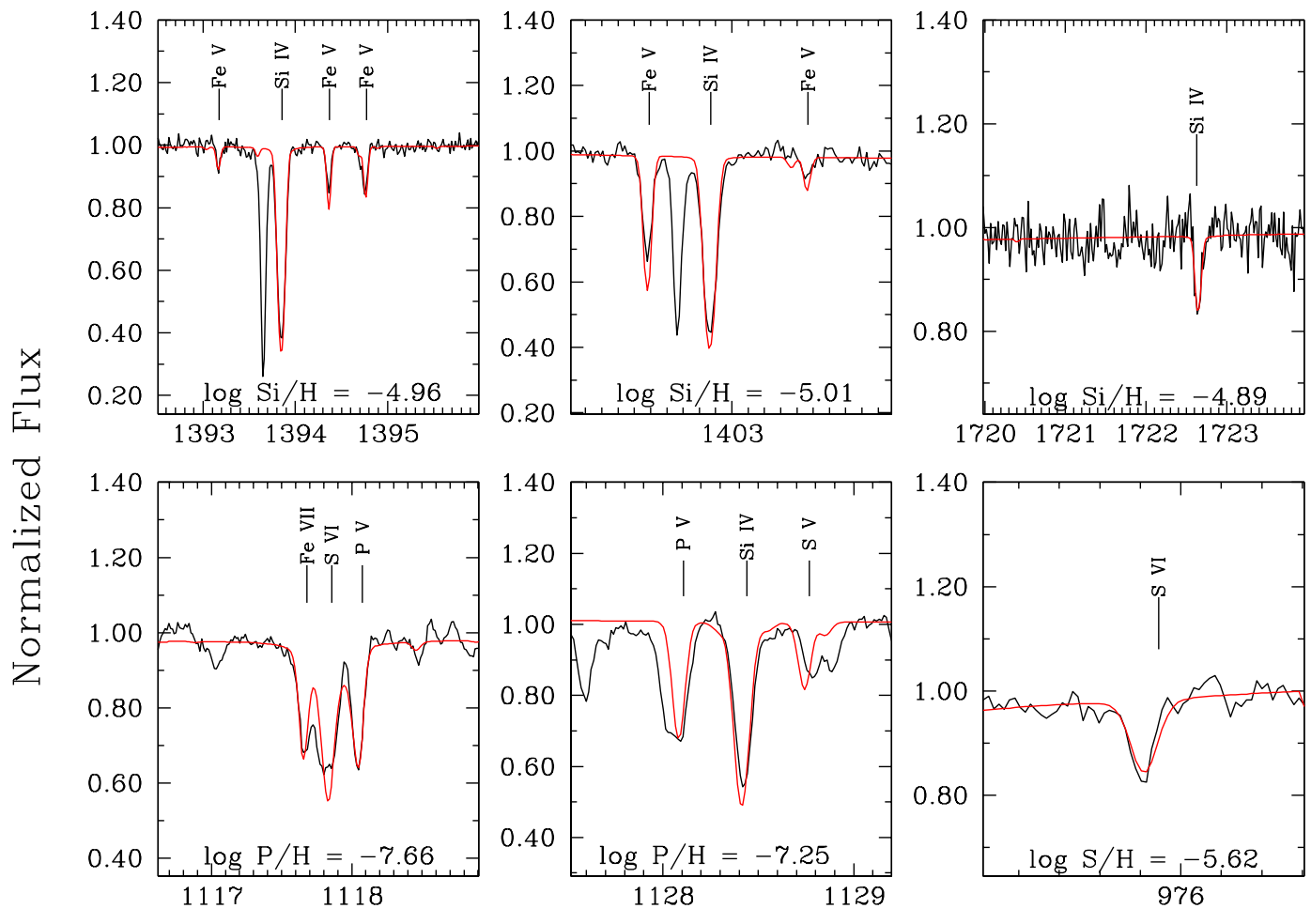

Wavelength $(\AA)$ 

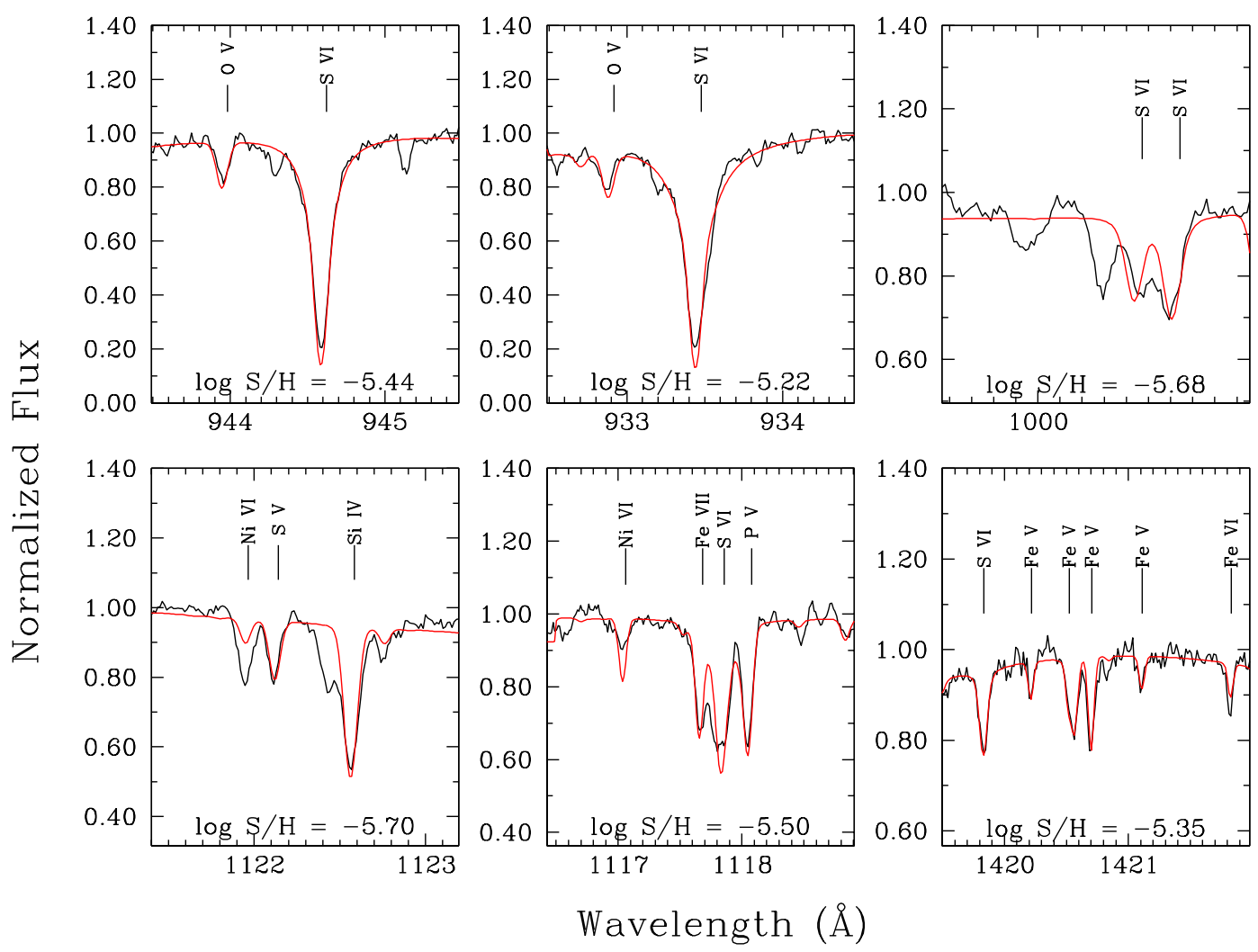

Online Figure panel e
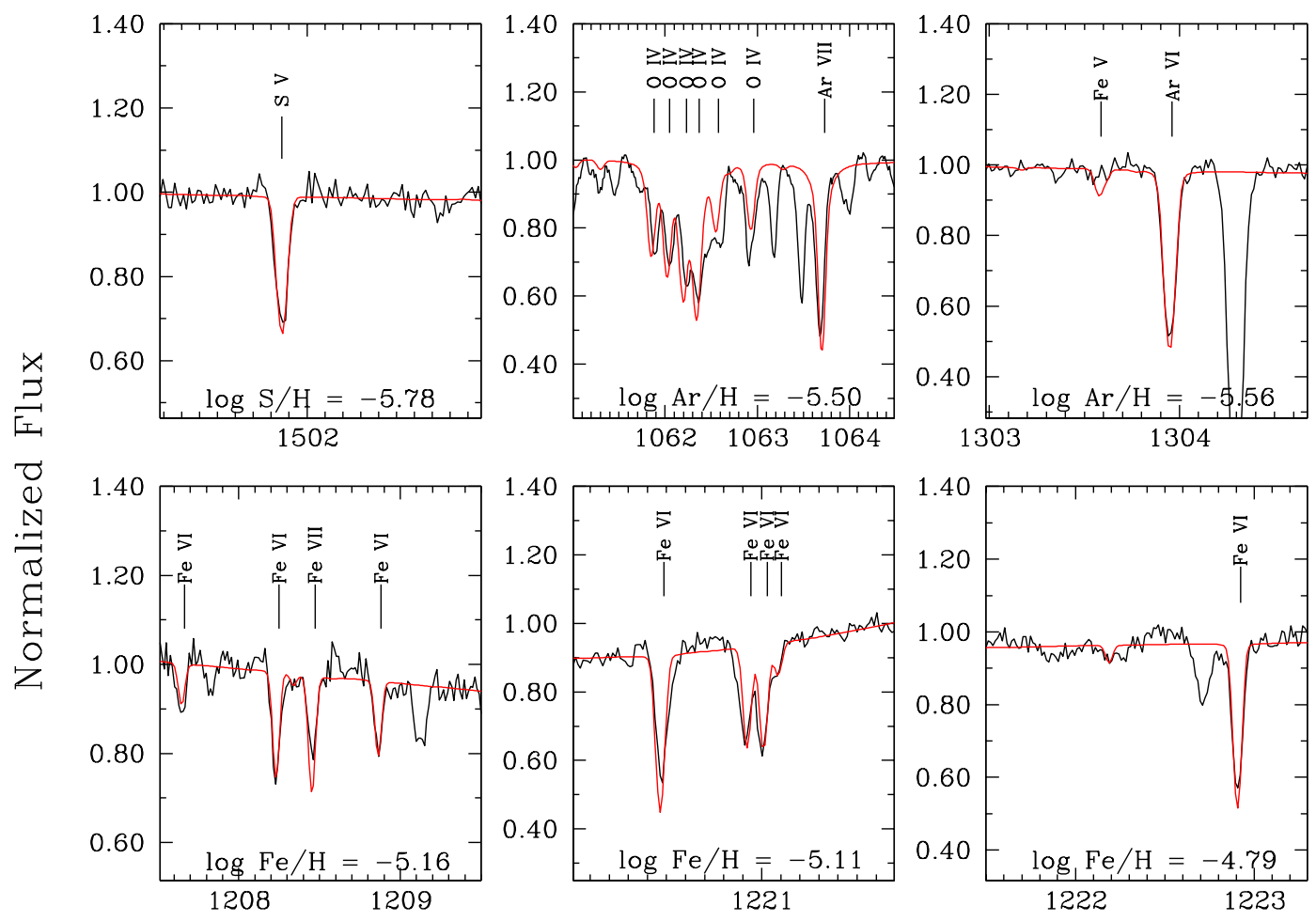

Wavelength $(\AA)$ 


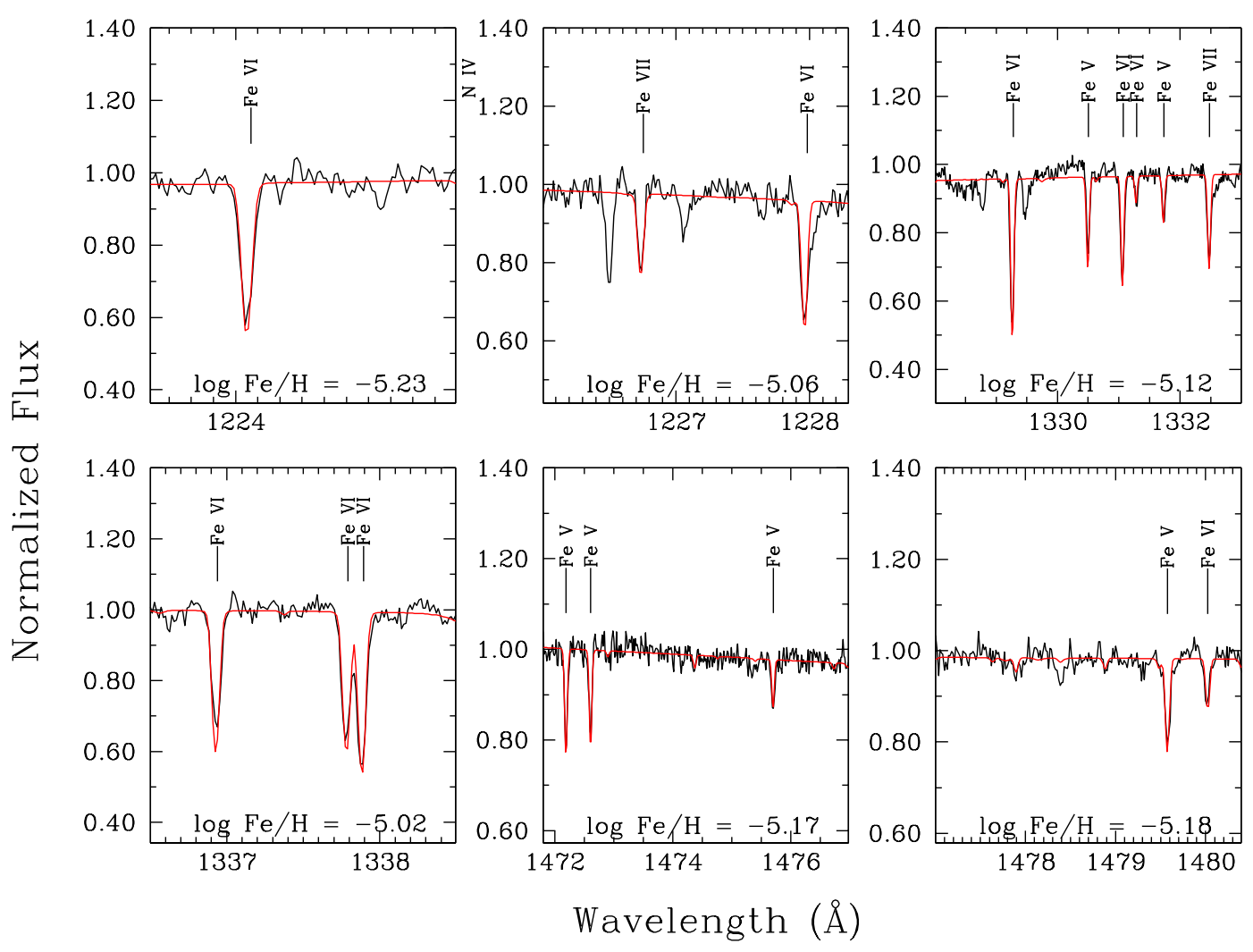

Online Figure panel g

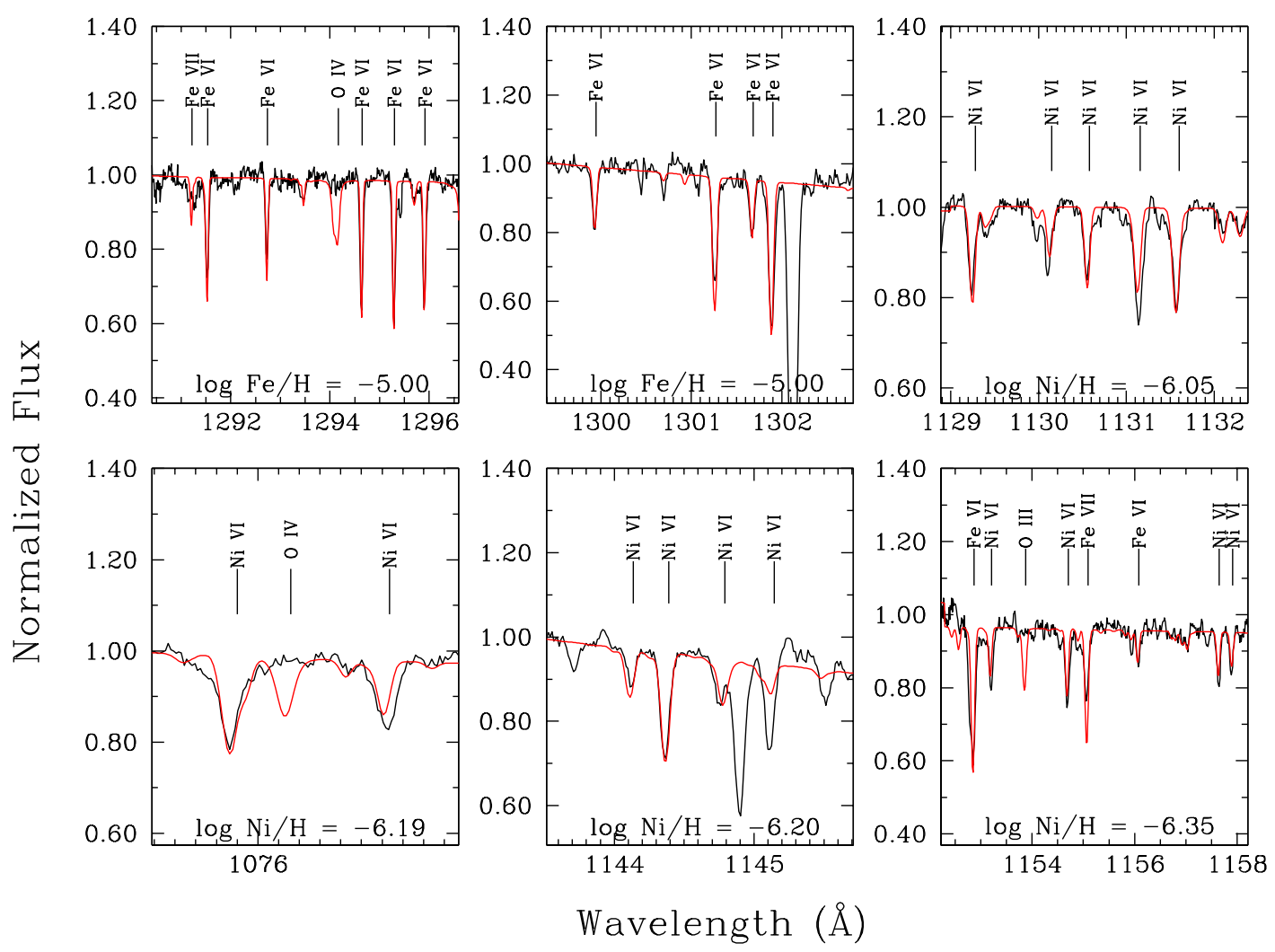

Online Figure panel h 


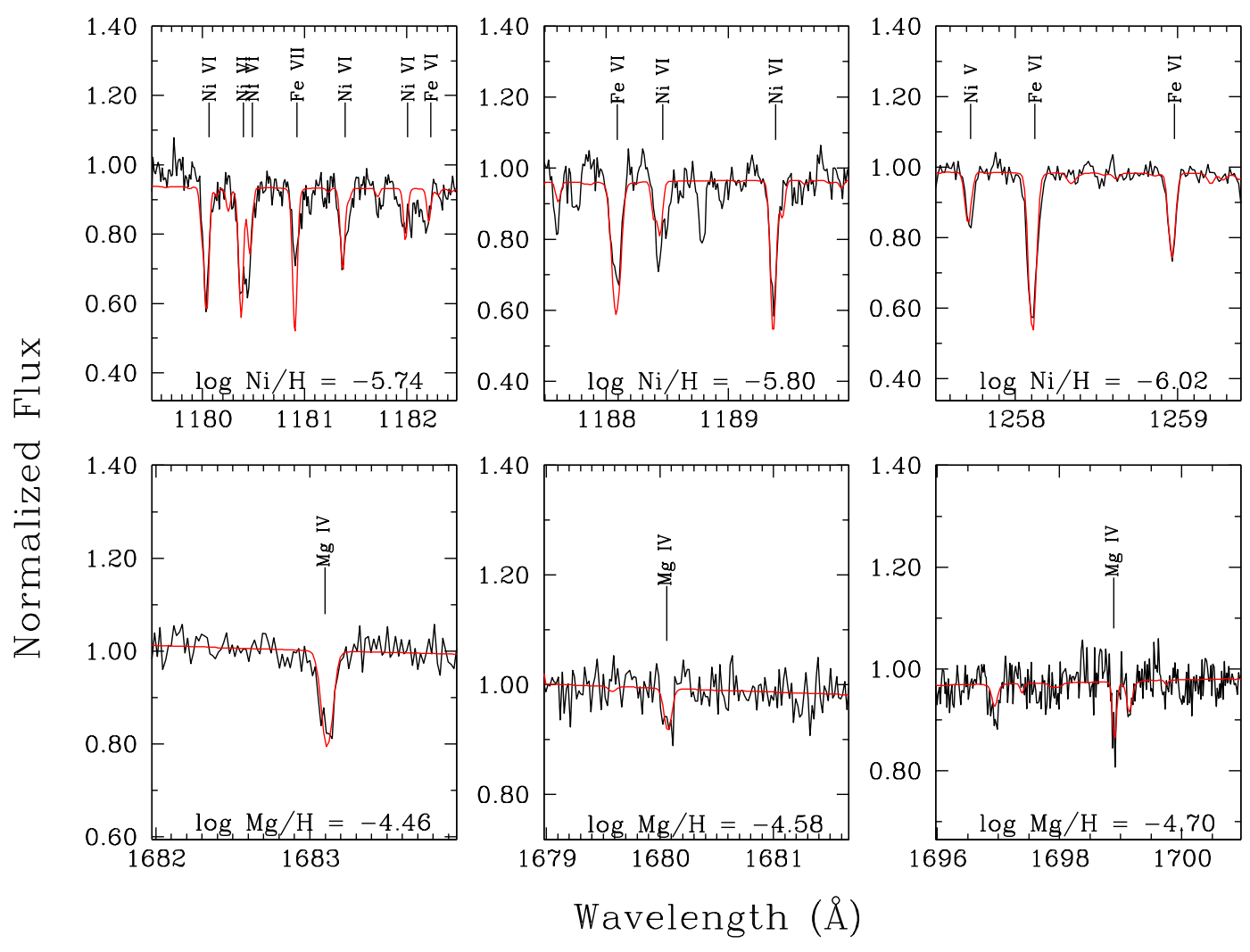

Online Figure panel i

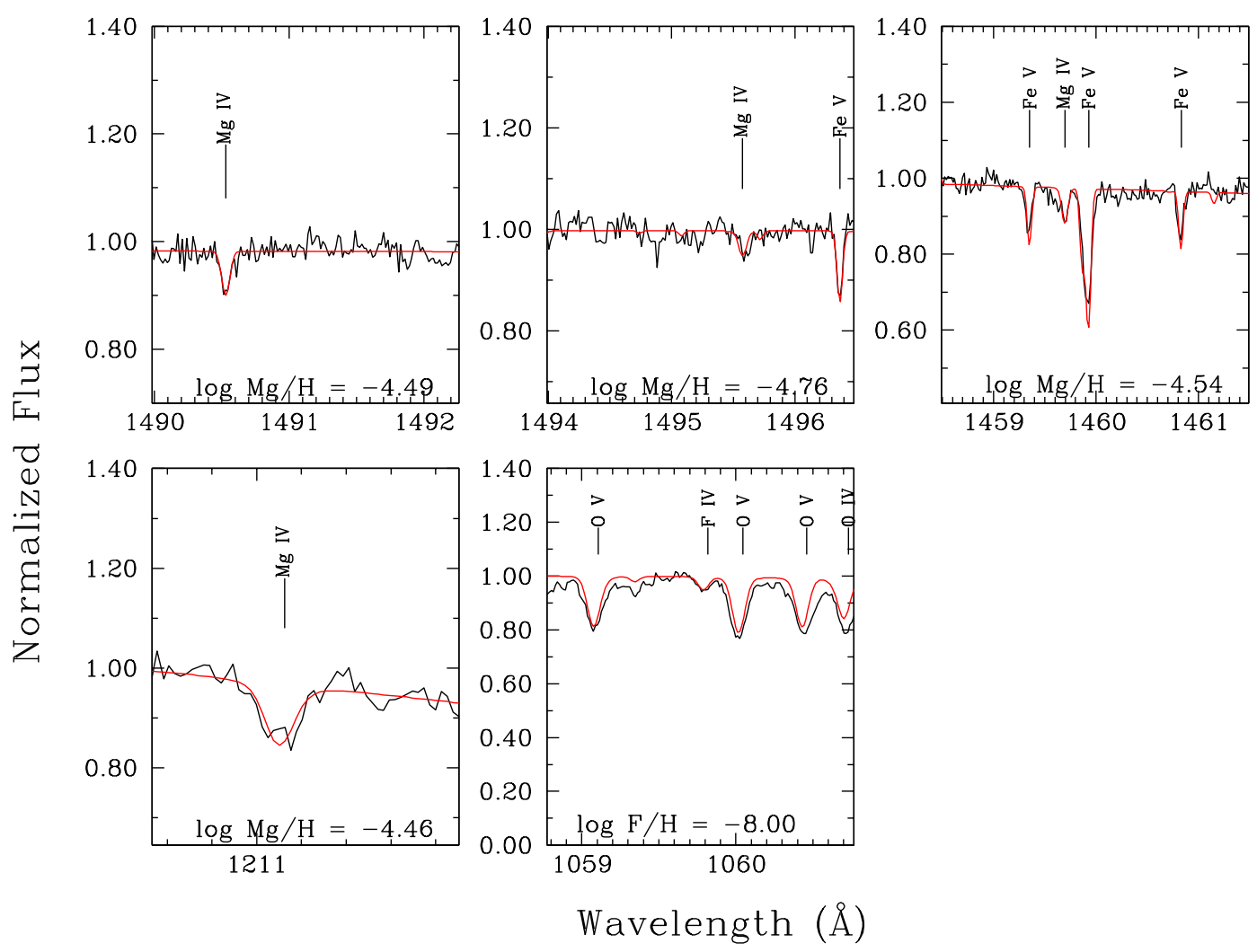

Online Figure panel j 